Pacific

Journal of

Mathematics

HOLOMORPHIC MAPS OF REAL SUBMANIFOLDS IN COMPLEX SPACES OF DIFFERENT DIMENSIONS

BERNHARD LAMEL 


\title{
HOLOMORPHIC MAPS OF REAL SUBMANIFOLDS IN COMPLEX SPACES OF DIFFERENT DIMENSIONS
}

\author{
BERNHARD LAMEL
}

\begin{abstract}
We define a new local invariant (called degeneracy) associated to a triple $\left(M, M^{\prime}, H\right)$, where $M \subset \mathbb{C}^{N}$ and $M^{\prime} \subset \mathbb{C}^{N^{\prime}}$ are real submanifolds of $\mathbb{C}^{N}$ and $\mathbb{C}^{N^{\prime}}$, respectively, and $H$ : $M \rightarrow M^{\prime}$ is either a holomorphic map, a formal holomorphic map, or a smooth CR-map. We use this invariant to find sufficient conditions under which finite jet dependence, convergence and algebraicity results hold.
\end{abstract}

\section{Introduction and statement of results.}

In this paper, we discuss mappings of generic real submanifolds in complex spaces of different dimensions. We address the following specific problems:

- Give conditions which ensure that a mapping depends on its finite jet.

- Give conditions under which a formal mapping between real-analytic generic submanifolds is convergent.

- Give conditions under which a map between algebraic submanifolds is algebraic.

The first two questions have attracted considerable attention in the equidimensional case, and quite complete results have been obtained for the class of finitely nondegenerate manifolds (see [2], [4]), and more recently, for target manifolds of finite type in the sense of D'Angelo $([\mathbf{9}])$ in $[\mathbf{3}]$. Whether similar results hold for mappings of generic submanifolds of spaces of different dimension is an intriguing problem which leads to some new geometric notions. The third question has also been answered in terms of characterizing the algebraic manifolds on which every holomorphic map is algebraic (see especially $[\mathbf{1 8}]$ and $[\mathbf{1}],[\mathbf{6}],[\mathbf{7}],[\mathbf{1 1}],[\mathbf{1 7}],[\mathbf{1 3}],[\mathbf{1 6}],[\mathbf{1 4}]$ ). We give a new sufficient condition, which can be checked using finitely many derivatives. For the definitions of a generic and a CR-manifold as well as other basic definitions, we refer the reader to e.g., [5].

Our starting point is the notion of $\left(k_{0}, s\right)$-degeneracy. This is a local invariant associated to the triple $\left(M, M^{\prime}, H\right)$, where $M \subset \mathbb{C}^{N}$ and $M^{\prime} \subset \mathbb{C}^{N^{\prime}}$ are generic $C^{\infty}$-submanifolds of $\mathbb{C}^{N}$ and $\mathbb{C}^{N^{\prime}}$, respectively, through 0 , and $H: M \rightarrow M^{\prime}$ is a map (for example, $C^{\infty}-\mathrm{CR}$ ) which in loose terms measures how "flat" $H(M)$ is as a submanifold of $M^{\prime} \subset \mathbb{C}^{N^{\prime}}$. The numbers $k_{0}$ and $s$ 
can be defined (at 0) as follows: If $\rho_{1}^{\prime}, \ldots \rho_{d^{\prime}}^{\prime}$ are defining functions for $M^{\prime}$, $L_{1}, \ldots, L_{n}$ is a local basis for the CR-vector fields on $M$, and $H(0)=0$, then

$$
N^{\prime}-s=\max _{k} \operatorname{dim}_{\mathbb{C}} \operatorname{span}_{\mathbb{C}}\left\{\left.L^{\alpha} \rho_{j, Z^{\prime}}^{\prime}(H(Z), \overline{H(Z)})\right|_{Z=0}:|\alpha| \leq k, 1 \leq j \leq d^{\prime}\right\},
$$

where for a multi-index $\alpha \in \mathbb{N}^{n}$ we write $L^{\alpha}=L_{1}^{\alpha_{1}} \cdots L_{n}^{\alpha_{n}}$, and $k_{0}$ is the least integer $k$ for which the maximum dimension on the right hand side of (1) is realized. Here we write $N=n+d$, where $d$ is the codimension of $M$; similarly, we shall write $N^{\prime}=n^{\prime}+d^{\prime}$, where $d^{\prime}$ is the codimension of $M^{\prime}$. An extension of this definition is given in Section 2 in the context of formal submanifolds and formal maps, which allows us a unified treatment of realanalytic and smooth manifolds. This new notion is related to the concept of finite nondegeneracy of a real submanifold (which was introduced for hypersurfaces in [6]), and we explore this relationship further in 2.4.

Particularly satisfying is the situation for mappings for which $s=0$. We call such mappings "nondegenerate", or more specifically, $k_{0}$-nondegenerate. These maps fulfill a sufficient condition to give a positive answer to all three points above; for example, every CR-diffeomorphism of class $C^{k_{0}}$ of $\left(k_{0^{-}}\right)$finitely nondegenerate submanifolds of $\mathbb{C}^{N}$, as introduced for hypersurfaces by Baouendi, Huang and Rothschild [6] (we will define those in Section 2) is a $k_{0}$-nondegenerate map. The other maps allowing for a further treatment are the ones which are of constant degeneracy (to be defined in Section 2 as well).

Let us recall that a formal holomorphic map $H=\left(H_{1}, \ldots, H_{N^{\prime}}\right): \mathbb{C}^{N} \rightarrow$ $\mathbb{C}^{N^{\prime}}$ at a point $p_{0}$ is an $N^{\prime}$-tuple of formal power series $H_{j}(Z)=\sum_{\alpha} c_{\alpha}^{j}(Z-$ $\left.p_{0}\right)^{\alpha}$, and if $H\left(p_{0}\right)=p_{0}^{\prime} \in \mathbb{C}^{N^{\prime}}$, we write $H:\left(\mathbb{C}^{N}, p_{0}\right) \rightarrow\left(\mathbb{C}^{N^{\prime}}, p_{0}^{\prime}\right)$ for such a map. If $p_{0} \in M, p_{0}^{\prime} \in M^{\prime}$ then we say that $H:\left(\mathbb{C}^{N}, p_{0}\right) \rightarrow\left(\mathbb{C}^{N^{\prime}}, p_{0}^{\prime}\right)$ maps $M$ into $M^{\prime}$ if the following property is satisfied: If $\rho^{\prime}=\left(\rho_{1}^{\prime}, \ldots, \rho_{d^{\prime}}^{\prime}\right)$ is a defining function of $M^{\prime}$ and $\rho=\left(\rho_{1}, \ldots, \rho_{d}\right)$ is a defining function of $M$ (where $d$ and $d^{\prime}$ are the codimensions of $M$ and $M^{\prime}$, respectively), then there is a $d^{\prime} \times d$ matrix $A$ of formal power series such that $\rho^{\prime}(H(Z), \bar{H}(\zeta))=A(Z, \zeta) \rho(Z, \zeta)$. (Here we are abusing notation: This equation shall hold in the sense of Taylor series.)

Let us recall that we say that $M$ is of finite type at $p$ (in the sense of Kohn-Bloom-Graham) if the CR and the anti-CR vectors together with their commutators of all length span the complexified tangent space of $M$ at $p$. We prove the following theorems. If not stated explicitly otherwise, all submanifolds are assumed to be smooth and connected.

Theorem 1. Let $M, M^{\prime}$ be generic real-analytic submanifolds of $\mathbb{C}^{N}$ and $\mathbb{C}^{N^{\prime}}$, respectively, $p_{0} \in M, M$ of finite type at $p_{0}, p_{0}^{\prime} \in M^{\prime}$, and assume that $H:\left(\mathbb{C}^{N}, p_{0}\right) \rightarrow\left(\mathbb{C}^{N^{\prime}}, p_{0}^{\prime}\right)$ is a formal holomorphic map which maps $M$ into 
$M^{\prime}$ and is $k_{0}$-nondegenerate at $p_{0}$. Then there exists a neighbourhood $U$ of $p_{0}$ in $\mathbb{C}^{N}$ on which $H$ is convergent.

For the next theorem, we denote by $j_{p_{0}}^{k} f$ the $k$-jet of $f$ at $p_{0}$.

Theorem 2. Let $M, M^{\prime}$ be generic real submanifolds of $\mathbb{C}^{N}$ and $\mathbb{C}^{N^{\prime}}$, respectively, $p_{0} \in M$, and $M$ of finite type at $p_{0}$. There exists an integer $K$ such that if $H: M \rightarrow M^{\prime}$ and $H^{\prime}: M \rightarrow M^{\prime}$ are $C^{\infty}{ }_{-} \mathrm{CR}$ mappings which are $k_{0}$-nondegenerate at $p_{0} \in M$ and $j_{p_{0}}^{K k_{0}} H=j_{p_{0}}^{K k_{0}} H^{\prime}$, then $j_{p_{0}}^{l} H=j_{p_{0}}^{l} H^{\prime}$ for all $l$.

Theorem 3. Let $M, M^{\prime}$ be generic real submanifolds of $\mathbb{C}^{N}$ and $\mathbb{C}^{N^{\prime}}$, respectively, $p_{0} \in M$, such that $M$ is of finite type at $p_{0}$. There exists an integer $K$ such that if $H: U \rightarrow \mathbb{C}^{N^{\prime}}$ is a holomorphic map defined on some neighbourhood $U$ of $p_{0}$ with $H(U \cap M) \subset M^{\prime}$ and such that $H$ is $k_{0}$-nondegenerate at $p_{0}$, and $H^{\prime}$ is another holomorphic map defined on some neighbourhood $U^{\prime}$ of $p_{0}$ with $H^{\prime}\left(U^{\prime} \cap M\right) \subset M^{\prime}$ with

$$
\frac{\partial^{\alpha} H}{\partial Z^{\alpha}}\left(p_{0}\right)=\frac{\partial^{\alpha} H^{\prime}}{\partial Z^{\alpha}}\left(p_{0}\right), \quad|\alpha| \leq K k_{0},
$$

then $H=H^{\prime}$.

Theorem 3 is an immediate consequence of Theorem 2. The proof of Theorem 1 and Theorem 2 is given in Section 3. Theorem 3 together with the reflection principle in [12] yields the following.

Corollary 4. Let $M, M^{\prime}$ be generic real-analytic submanifolds of $\mathbb{C}^{N}$ and $\mathbb{C}^{N^{\prime}}$, respectively, $p_{0} \in M$, and $M$ of finite type at $p_{0}$. There exists an integer $K$ such that if $H: M \rightarrow M^{\prime}$ and $H^{\prime}: M \rightarrow M^{\prime}$ are $C^{K k_{0}}{ }_{-} \mathrm{CR}$ mappings which are $k_{0}$-nondegenerate at $p_{0} \in M$ and $j_{p_{0}}^{K k_{0}} H=j_{p_{0}}^{K k_{0}} H^{\prime}$, then both extend to holomorphic mappings and $H=H^{\prime}$.

Note that the notion of nondegeneracy makes sense even for maps which are a priori only smooth up to a certain finite order, so that the statement of this corollary makes sense. The last result we prove about nondegenerate maps is an algebraicity theorem.

Theorem 5. Let $M$ and $M^{\prime}$ be algebraic generic submanifolds of $\mathbb{C}^{N}$ and $\mathbb{C}^{N^{\prime}}$, respectively, $H$ a holomorphic map defined on some connected neighbourhood $U$ of $M$ with $H(M) \subset M^{\prime}$ and such that $H$ is $k_{0}$-nondegenerate at some point of $M$. Then $H$ is algebraic.

Our next results are for hypersurfaces. They are valid either in the setting where $N^{\prime}=N+1$, and the hypersurfaces are assumed to be Levinondegenerate, or, where the target hypersurface is strictly pseudoconvex and the source hypersurface is of finite type (and there are no restrictions on $N^{\prime}$ ). In the case of Levi-nondegenerate hypersurfaces, we will consider 
maps $H$ which are (CR) transversal (the formal definition of this property is given in Definition 19). We will refer to the following properties in the theorems below:

(P1) $M^{\prime}$ is strictly pseudoconvex at $p_{0}^{\prime}$.

(P2) $N^{\prime}=N+1$ and $M$ and $M^{\prime}$ are Levi-nondegenerate at $p_{0}$ and $p_{0}^{\prime}$, respectively, and $H$ is transversal at $p_{0}$.

Theorem 6. Let $M, M^{\prime}$ be real-analytic hypersurfaces in $\mathbb{C}^{N}$ and $\mathbb{C}^{N^{\prime}}$, respectively, $p_{0} \in M, p_{0}^{\prime} \in M^{\prime}, M$ of finite type at $p_{0}$, and let $H:\left(\mathbb{C}^{N}, p_{0}\right) \rightarrow$ $\left(\mathbb{C}^{N^{\prime}}, p_{0}^{\prime}\right)$ be a formal holomorphic map of constant degeneracy which maps $M$ into $M^{\prime}$. Then there exists a neighbourhood $U$ of $p_{0}$ in $\mathbb{C}^{N}$ on which $H$ is convergent given that either (P1) or (P2) holds.

Note that the case $N^{\prime}=N+1$ is very special, as the following example shows.

Example 1. Let $M \subset \mathbb{C}^{N}$ be given by $\operatorname{Im} w=\sum_{j=1}^{n}\left|z_{j}\right|^{2}$, and $M^{\prime} \subset \mathbb{C}^{N+2}$ be given by $\operatorname{Im} w^{\prime}=\left|z_{n+2}\right|^{2}-\sum_{j=1}^{n+1}\left|z_{j}^{\prime}\right|^{2}$ ("adding a black hole"). Then the $\operatorname{map}\left(z_{1}, \ldots, z_{n}, w\right) \mapsto\left(z_{1}, \ldots, z_{n}, f(z, w), f(z, w), w\right)$ maps $M$ into $M^{\prime}$ for every (formal) holomorphic map $f: \mathbb{C}^{N} \rightarrow \mathbb{C}$.

This example also shows that in general, algebraicity and dependence on jets of finite order for Levi-nondegenerate hypersurfaces can only be expected in the case $N^{\prime}=N+1$ (without further restrictions on the mappings, as for example nondegeneracy as introduced above).

Theorem 7. Let $M, M^{\prime}$ be real hypersurfaces in $\mathbb{C}^{N}$ and $\mathbb{C}^{N^{\prime}}$, respectively, $p_{0} \in M$, and $M$ of finite type at $p_{0}$. If $H: M \rightarrow M^{\prime}$ and $H^{\prime}: M \rightarrow M^{\prime}$ are $C^{\infty}$ _CR mappings which are constantly $\left(k_{0}, s\right)$-degenerate near $p_{0} \in M$ with $j_{p_{0}}^{2 k_{0}} H=j_{p_{0}}^{2 k_{0}} H^{\prime}$, then $j_{p_{0}}^{l} H=j_{p_{0}}^{l} H^{\prime}$ for all $l$, provided that either $(\mathrm{P} 1)$ or (P2) holds.

Theorem 8. Let $M, M^{\prime}$ be real hypersurfaces in $\mathbb{C}^{N}$ and $\mathbb{C}^{N^{\prime}}$, respectively, $p_{0} \in M$, and $M$ of finite type at $p_{0}$. If $H: U \rightarrow \mathbb{C}^{N^{\prime}}$ is a holomorphic map defined on some neighbourhood $U$ of $p_{0}$ with $H(U \cap M) \subset M^{\prime}$ which is constantly $\left(k_{0}, s\right)$-degenerate at $p_{0}$, and $H^{\prime}$ is another holomorphic map defined on some neighbourhood $U^{\prime}$ of $p_{0}$ with $H^{\prime}\left(U^{\prime} \cap M\right) \subset M^{\prime}$ with

$$
\frac{\partial^{\alpha} H}{\partial Z^{\alpha}}\left(p_{0}\right)=\frac{\partial^{\alpha} H^{\prime}}{\partial Z^{\alpha}}\left(p_{0}\right), \quad|\alpha| \leq 2 k_{0},
$$

then $H=H^{\prime}$, provided that either (P1) or (P2) holds.

We also have the following algebraicity result. Case (i) below is actually contained in the results in [18].

Theorem 9. Let $M$ and $M^{\prime}$ be algebraic hypersurfaces in $\mathbb{C}^{N}$ and $\mathbb{C}^{N^{\prime}}$, respectively, $H$ a holomorphic map defined on some connected neighbourhood 
$U$ of $M$ with $H(U \cap M) \subset M^{\prime}$. Then $H$ is algebraic, provided that either of the following additional properties hold:

(i) There exists a point $p_{0}$ in $M$ where $M$ is of finite type and $M^{\prime}$ is strictly pseudoconvex at $H\left(p_{0}\right)$;

(ii) $N^{\prime}=N+1$, and there exists a point $p_{0} \in M$ at which $H$ is transversal, and $M$ and $M^{\prime}$ are Levi-nondegenerate at $p_{0}$ and $H\left(p_{0}\right)$, respectively.

Theorem 8 is again an immediate consequence of Theorem 7 . In the case $N^{\prime}=N+1$, with the assumptions of the theorem, $s=0$ or $s=1$ (see Lemma 20); the case $s=0$ is covered by Theorems 2 and 3. The proofs of Theorems 6, 9 and 7 in the Levi-nondegenerate case are given in Section 4. The proof for strictly pseudoconvex hypersurfaces is given in Section 5 .

Acknowledgements. The author thanks the referee for many helpful comments and suggestions as well as for pointing out many typographical errors. Furthermore, the author would like to thank S. Baouendi and L. Rothschild for their help and their interest in this work.

\section{Formal holomorphic maps of constant degeneracy.}

2.1. Some definitions. In this section we want to give a short review of some basic definitions. We will be very brief, focusing mostly on the facts which we shall need later on. The purpose of this section is mainly for reference. A thorough discussion of the definitions given here can be found in e.g., [3].

2.1.1. Formal submanifolds and formal maps. Consider the ring of formal power series $\mathbb{C} \llbracket Z, \zeta \rrbracket$ in the $2 \mathrm{~N}$ indeterminates $(Z, \zeta)=\left(Z_{1}, \ldots, Z_{N}\right.$, $\left.\zeta_{1}, \ldots, \zeta_{N}\right)$. A formal generic submanifold $M \subset \mathbb{C}^{N}$ of codimension $d$ is an ideal $I \subset \mathbb{C} \llbracket Z, \zeta \rrbracket$ which can be generated by $d$ formal power series $\rho_{1}(Z, \zeta), \ldots, \rho_{d}(Z, \zeta)$ such that $\bar{\rho}_{j}(\zeta, Z) \in I, 1 \leq j \leq d$ and such that the vectors $\rho_{1, Z}(0), \ldots, \rho_{d, Z}(0)$ are linearly independent (where for $\phi \in \mathbb{C} \llbracket Z, \zeta \rrbracket$, $\phi_{Z}=\left(\phi_{Z_{1}}, \ldots, \phi_{Z_{N}}\right)$, and $\bar{\phi}$ denotes the power series with complex conjugated coefficients). A formal holomorphic change of coordinates $\widetilde{Z}=F(Z)$ is the ring isomorphism between $\mathbb{C} \llbracket \widetilde{Z}, \widetilde{\zeta} \rrbracket$ and $\mathbb{C} \llbracket Z, \zeta \rrbracket$ induced by $\widetilde{Z}_{j}=F_{j}(Z)$, $\widetilde{\zeta}_{j}=\bar{F}_{j}(\zeta)$, where $F=\left(F_{1}, \ldots, F_{N}\right)$ with $F_{j} \in \mathbb{C} \llbracket Z \rrbracket, 1 \leq j \leq N$, satisfying

$$
\operatorname{det}\left(\frac{\partial F_{j}}{\partial Z_{k}}(0)\right)_{\substack{1 \leq j \leq N \\ 1 \leq k \leq N}} \neq 0 .
$$

A formal holomorphic map $H: \mathbb{C}^{N} \rightarrow \mathbb{C}^{N^{\prime}}$ is an $N^{\prime}$-tuple of formal power series in $Z, H=\left(H_{1}, \ldots, H_{N^{\prime}}\right), H_{j} \in \mathbb{C} \llbracket Z \rrbracket$ for $1 \leq j \leq N^{\prime}$. A formal holomorphic map induces a ring homomorphism $H^{\sharp}: \mathbb{C} \llbracket Z^{\prime}, \zeta^{\prime} \rrbracket \rightarrow \mathbb{C} \llbracket Z, \zeta \rrbracket$ by $H^{\sharp}\left(Z_{j}^{\prime}\right)=H_{j}(Z), H^{\sharp}\left(\zeta_{j}^{\prime}\right)=\bar{H}_{j}(\zeta)$. Given two formal submanifolds $M \subset \mathbb{C}^{N}$ 
and $M^{\prime} \subset \mathbb{C}^{N^{\prime}}$, of codimension $d$ and $d^{\prime}$ respectively, represented by their ideals $I \subset \mathbb{C} \llbracket Z, \zeta \rrbracket$ and $I^{\prime} \subset \mathbb{C} \llbracket Z^{\prime}, \zeta^{\prime} \rrbracket$ we say that $H$ maps $M$ into $M^{\prime}$ if $H^{\sharp}\left(I^{\prime}\right) \subset I$. (From now on we will write $H: M \rightarrow M^{\prime}$ to indicate that we are in this situation.) This is the case if and only if for any generators $\rho=\left(\rho_{1}, \ldots, \rho_{d}\right)$ of $I$ and generators $\rho^{\prime}=\left(\rho_{1}^{\prime}, \ldots, \rho_{d^{\prime}}^{\prime}\right)$ we have that

$$
\rho^{\prime}(H(Z), \bar{H}(\zeta))=A(Z, \zeta) \rho(Z, \zeta),
$$

where $A(Z, \zeta)$ is a $d^{\prime} \times d$ matrix of formal power series in $(Z, \zeta)$. If in addition $d=d^{\prime}$ and $H$ is a formal holomorphic change of coordinates, then $\operatorname{det} A(0,0) \neq 0$, so that $A$ is an invertible matrix of formal power series.

We work with formal power series since if we want to handle $C^{\infty}$-submanifolds, then they are a convenient way of keeping track of all equations which we arrive from by repeated differentiation. This brings us to the subject of formal vector fields. A formal vector field $X$ is an operator of the form

$$
X=\sum_{j=1}^{N} a_{j}(Z, \zeta) \frac{\partial}{\partial Z_{j}}+\sum_{j=1}^{N} b_{j}(Z, \zeta) \frac{\partial}{\partial \zeta_{j}},
$$

where $a_{j}(Z, \zeta)$ and $b_{j}(Z, \zeta)$ are formal power series. One checks that the formal vector fields are exactly the derivations of the $\mathbb{C}$-algebra $\mathbb{C} \llbracket Z, \zeta \rrbracket . X$ is of type $(1,0)$ if $b_{j}(Z, \zeta)=0,1 \leq j \leq N$, and of type $(0,1)$ if $a_{j}(Z, \zeta)=0$, $1 \leq j \leq N$, and this distinction is invariant under formal holomorphic changes of coordinates. A formal vector field is tangent to $M$ if $X I \subset I$ (as above, $I$ denotes the ideal representing $M$ ).

A formal CR-vector field tangent to $M$ is a formal vector field of type $(0,1)$ tangent to $M$. We write $\mathcal{D}_{M}^{0,1}$ for the formal CR-vector fields tangent to $M$. This is a $\mathbb{C} \llbracket Z, \zeta \rrbracket$-module. Finally, we say that $L_{1}, \ldots, L_{n}($ where $n=N-d)$ is a basis of the formal CR-vector fields tangent to $M$ if they generate the quotient module $\mathcal{D}_{M}^{0,1} / I \mathcal{D}_{M}^{0,1}$. For the anti-CR-vector fields tangent to $M$ (that is, the formal vector fields of type $(1,0)$ tangent to $M$ ) we write $\mathcal{D}_{M}^{1,0}$, and set $\mathcal{D}_{M}=\mathcal{D}_{M}^{0,1} \oplus \mathcal{D}_{M}^{1,0}$. For the Lie algebra generated by $\mathcal{D}_{M}$, we write $\mathfrak{g}$. We say that $M$ is of finite type (at 0 ) if $\operatorname{dim}_{\mathbb{C}} \mathfrak{g}(o)=2 n+d$.

2.1.2. Formal normal coordinates. Let $M$ be a generic formal submanifold of codimension $d$. Then after a formal holomorphic change of coordinates we can assume $Z=(z, w)=\left(z_{1}, \ldots, z_{n}, w_{1}, \ldots, w_{d}\right) \in \mathbb{C}^{n} \times \mathbb{C}^{d}$ (for the corresponding $\zeta$ we write $\zeta=(\chi, \tau))$ and that $I$ is generated by $d$ functions $w_{j}-Q_{j}(z, \chi, \tau), j=1, \ldots, d$, where $Q_{j} \in \mathbb{C} \llbracket z, \chi, \tau \rrbracket$ fulfills

$$
Q_{j}(z, 0, \tau)=Q_{j}(0, \chi, \tau)=\tau_{j}, \quad j=1, \ldots, d .
$$

In that case, another useful set of generators is given by $\tau_{j}=\bar{Q}_{j}(\chi, z, w)$, $j=1, \ldots, d$. We shall call such coordinates formal normal coordinates. We 
can use them in order to parametrize $M$ : Under this, we will understand the ring isomorphism

$$
\mathbb{C} \llbracket z, w, \chi, \tau \rrbracket / I \rightarrow \mathbb{C} \llbracket z, \chi, \tau \rrbracket, \quad z \mapsto z, w \mapsto Q(z, \chi, \tau), \chi \mapsto \chi, \tau \mapsto \tau,
$$

or

(9) $\mathbb{C} \llbracket z, w, \chi, \tau \rrbracket / I \rightarrow \mathbb{C} \llbracket \chi, z, w \rrbracket, \quad z \mapsto z, w \mapsto w, \chi \mapsto \chi, \tau \mapsto \bar{Q}(\chi, z, w)$.

Note that a basis of CR-vector fields tangent to $M$ is given by

$$
L_{k}=\frac{\partial}{\partial \chi_{k}}+\sum_{j=1}^{d} \bar{Q}_{j \chi_{k}}(\chi, z, w) \frac{\partial}{\partial \tau_{j}}, \quad k=1, \ldots, n .
$$

For a multi-index $\alpha=\left(\alpha_{1}, \ldots, \alpha_{n}\right) \in \mathbb{N}^{n}$ we write $L^{\alpha}=L_{1}^{\alpha_{1}} \cdots L_{n}^{\alpha_{n}}$. Let now $\phi(z, w, \chi, \tau)$ be a formal power series. We want to relate the image of $\phi$ in the parametrization of $M$ given by (9) with its derivatives along CR-directions. Expand $\phi$ as a series in $\mathbb{C} \llbracket z, w \rrbracket \llbracket \chi \rrbracket$ :

$$
\phi(z, w, \chi, \bar{Q}(\chi, z, w))=\sum_{\alpha \in \mathbb{N}^{n}} \frac{s_{\alpha}(z, w)}{\alpha !} \chi^{\alpha} .
$$

Now the $s_{\alpha}$ are obtained by partial differentiation:

$$
s_{\alpha}(z, w)=\left.\frac{\partial^{|\alpha|}}{\partial \chi^{\alpha}} \phi(z, w, \chi, \bar{Q}(\chi, z, w))\right|_{\chi=0}=L^{\alpha} \phi(z, w, 0, w) .
$$

The last equality is proved by induction on $|\alpha|$ and its proof is left to the reader.

2.1.3. Segre-mapppings and a finite type criterion. Again, we are considering a generic formal submanifold $M \subset \mathbb{C}^{N}$ of codimension $d$. Assume that formal normal coordinates $(z, w)$ as in 2.1 .2 have been chosen, along with the corresponding (vector valued) function $Q(z, \chi, \tau) \in$ $\mathbb{C} \llbracket z, \chi, \tau \rrbracket^{d}$, fulfilling (7), such that $I$ (the ideal associated to $M$ ) is generated by $w_{j}-Q_{j}(z, \chi, \tau), j=1, \ldots, d$ (or, equivalently, by $\tau_{j}-\bar{Q}_{j}(\chi, z, w)$ ). The Segre mappings are the formal mappings $v^{k}: \mathbb{C}^{k n} \rightarrow \mathbb{C}^{N}$, for any integer $k$, defined by

$$
v^{0}=(0,0), \quad v^{1}(z)=(z, 0),
$$

$$
v^{2 j}\left(z, \chi^{1}, \ldots, z^{j-1}, \chi^{j}\right)=\left(z, Q\left(z, \chi^{1}, \bar{Q}\left(\chi^{1}, z^{1}, \ldots, Q\left(z^{j-1}, \chi^{j}, 0\right) \cdots\right)\right)\right),
$$

$$
v^{2 j+1}\left(z, \chi^{1}, \ldots, \chi^{j}, z^{j}\right)=\left(z, Q\left(z, \chi^{1}, \bar{Q}\left(\chi^{1}, z^{1}, \ldots, \bar{Q}\left(\chi^{j}, z^{j}, 0\right) \cdots\right)\right)\right) .
$$


We will write $\left(z, \chi^{1}, z^{1}, \ldots\right)=(z, \xi)$, where $\xi=\left(\chi^{1}, z^{1}, \ldots\right) \in \mathbb{C}^{(k-1) n}$. These mappings have the property that for every $k \geq 0$, and for every $f \in I$,

$$
f\left(v^{k+1}(z, \xi), \bar{v}^{k}(\xi)\right)=0 .
$$

We shall need the finite type criterion of Baouendi, Ebenfelt and Rothschild, which we state here for reference; see e.g., [3] or [5].

Let $F: \mathbb{C}^{p} \rightarrow \mathbb{C}^{r}$ be a formal mapping, that is, an $r$-tuple of formal power series in $p$ variables $\left(x_{1}, \ldots, x_{p}\right)$. We denote by $\operatorname{rk} F$ the rank of the Jacobian matrix of $F$ over the quotient field of the ring of formal power series $\mathbb{C} \llbracket x_{1}, \ldots, x_{p} \rrbracket$.

Theorem 10. Let $M$ be a formal generic submanifold of $\mathbb{C}^{N}$ of codimension $d$. Then, $M$ is of finite type at 0 if and only if there exists a $k_{1} \leq d+1$ such that $\operatorname{rk}\left(v^{k}\right)=N$ for $k \geq k_{1}$. Moreover, if $M$ is real-anlaytic and of finite type at 0 , then there exists $\left(z_{0}, \xi_{0}\right) \in \mathbb{C}^{n} \times \mathbb{C}^{\left(2 k_{1}-1\right) n}$ arbitrarily close to the origin such that $v^{2 k_{1}}\left(z_{0}, \xi_{0}\right)=0$ and the rank of the Jacobian matrix of $v^{2 k_{1}}$ at $\left(z_{0}, \xi_{0}\right)$ is $N$.

Note the following consequence for real-analytic submanifolds: There exist points $\left(z_{0}, \xi_{0}\right) \in \mathbb{C}^{n} \times \mathbb{C}^{2 k_{1}}$, arbitrarily close to 0 , such that the function $v^{2 k_{1}}$ has a holomorphic right inverse $\psi: \mathbb{C}^{N} \rightarrow \mathbb{C}^{n} \times \mathbb{C}^{2 k_{1}}$ defined in a neighbourhood of $0 \in \mathbb{C}^{N}$, such that $\psi(0)=\left(z_{0}, \xi_{0}\right)$ and $v^{2 k}(\psi(Z))=Z$. This follows from the inverse function theorem and Theorem 10.

2.2. Constant-rank submodules. We are now considering a free module of rank $k$ over $\mathbb{C} \llbracket Z, \zeta \rrbracket$. Let $E \subset \mathbb{C} \llbracket Z, \zeta \rrbracket^{k}$ be a submodule and $I \subset \mathbb{C} \llbracket Z, \zeta \rrbracket$ an ideal.

Definition 11. The submodule $E \subset \mathbb{C} \llbracket Z, \zeta \rrbracket^{k}$ is of constant rank $l=$ $\operatorname{dim}_{\mathbb{C}} E(0)$ over $I$ if for all $e_{1}, \ldots, e_{l}, e_{l+1} \in E$, where $e_{j}=\left(e_{j}^{1}, \ldots, e_{j}^{k}\right)$, every subminor of length $l+1$ of the matrix $\left(e_{m}^{n}\right)_{1 \leq m \leq l+1,1 \leq n \leq k}$ is an element of $I$.

Definition 11 is motivated by the following observation. Consider a smooth submanifold $M \subset \mathbb{C}^{N}$. By taking the Taylor series of the defining functions of $M$ at a point, one obtains a formal submanifold represented by some ideal $I$. Consider a vector bundle of rank $l$ over $M$, embedded in $\mathbb{C}^{k}$. Its sections will be a submodule of $\Gamma\left(M, \mathbb{C}^{k}\right) \cong C^{\infty}(M)^{k}$ (the isomorphism is determined by a choice of coordinates). Taking the Taylor expansion of the components, we get a submodule $E \subset \mathbb{C} \llbracket Z, \zeta \rrbracket^{k}$ which fulfills the condition of Definition 11 over $I$.

We may refer to a submodule of constant rank $l$ as a formal vector bundle over $M$. This is also highlighted by the following Lemma, which can be thought of as a characterization of the bases of sections of a formal vector bundle. 
Lemma 12. Suppose that $E$ is a submodule of $\mathbb{C} \llbracket Z, \zeta \rrbracket^{k}$, with $\operatorname{dim}_{\mathbb{C}} E(0)=$ l. Then $E$ is of constant rank $l$ over $I \subset \mathbb{C} \llbracket Z, \zeta \rrbracket$ if and only if $E$ has the following property: If $v_{1}, \ldots, v_{l} \in E$ are vectors such that $v_{1}(0), \ldots v_{l}(0)$ form a basis of $E(0)$, then $v_{1}, \ldots, v_{l}$ generate $E$ up to vectors all of whose components are elements of $I$.

Proof. The "if" direction is trivial; so assume that $E$ is of constant rank $l$. After reordering, we can assume that if we write $v_{j}=\left(v_{j}^{1}, \ldots, v_{j}^{k}\right)$, the matrix $\left(v_{m}^{n}\right)_{1 \leq m, n \leq l}$ is invertible. Hence, given any $e=\left(e^{1}, \ldots, e^{k}\right) \in E$, we can find $a_{1}, \ldots, a_{l} \in \mathbb{C} \llbracket Z, \zeta \rrbracket$ such that $\sum_{m=1}^{l} a_{n} v_{m}^{j}=e^{j}, j=1, \ldots, l$. Now consider $e^{\prime}=e-\sum_{j} a_{j} v_{j} \in E$. We want to show that the components of this vector are elements of $I$; this is clear for the first $l$ components (which are 0 , after all). Taking a subminor of length $l+1$ of the matrix

$$
\left(\begin{array}{ccccc}
v_{1}^{1} & \ldots & v_{1}^{l} & \ldots & v_{1}^{k} \\
\vdots & & \vdots & & \vdots \\
v_{l}^{1} & \ldots & v_{l}^{l} & \ldots & v_{l}^{k} \\
e^{\prime 1} & \ldots & e^{\prime l} & \ldots & e^{\prime k}
\end{array}\right)
$$

which contains the first $l$ columns and developing it along the last row, by assumption we have that

$$
\pm\left|\begin{array}{ccc}
v_{1}^{1} & \ldots & v_{1}^{l} \\
\vdots & & \vdots \\
v_{l}^{1} & \ldots & v_{l}^{l}
\end{array}\right| e^{\prime j} \in I, \quad l+1 \leq j \leq k
$$

which implies $e^{\prime j} \in I$ since the determinant in (16) is a unit in $\mathbb{C} \llbracket Z, \zeta \rrbracket$.

2.3. Degeneracy of a formal holomorphic map. Let $H: M \rightarrow M^{\prime}$ be a formal holomorphic map between the formal submanifolds $M \subset \mathbb{C}^{N}$ and $M^{\prime} \subset \mathbb{C}^{N^{\prime}}$. Choose a basis of formal CR-vector fields $L_{1}, \ldots, L_{n}$ tangent to $M$ and generators $\rho_{1}^{\prime}\left(Z^{\prime}, \zeta^{\prime}\right), \ldots, \rho_{d^{\prime}}^{\prime}\left(Z^{\prime}, \zeta^{\prime}\right)$ of the ideal $I^{\prime}$ representing $M^{\prime}$. The formal series $\rho_{1}^{\prime}(H(Z), \bar{H}(\zeta)), \ldots, \rho_{d^{\prime}}^{\prime}(H(Z), \bar{H}(\zeta))$ are then elements of $I$ (since $\left.H: M \rightarrow M^{\prime}\right)$. For $\rho_{j}^{\prime}\left(Z^{\prime}, \zeta^{\prime}\right)$, we denote by $\rho_{j, Z^{\prime}}^{\prime}\left(Z^{\prime}, \zeta^{\prime}\right)$ the complex gradient

$$
\rho_{j, Z^{\prime}}^{\prime}\left(Z^{\prime}, \zeta^{\prime}\right)=\left(\frac{\partial \rho_{j}^{\prime}\left(Z^{\prime}, \zeta^{\prime}\right)}{\partial Z_{1}^{\prime}}, \ldots, \frac{\partial \rho_{j}^{\prime}\left(Z^{\prime}, \zeta^{\prime}\right)}{\partial Z_{N^{\prime}}^{\prime}}\right)
$$

and we usually think of it as a row vector. So $\rho_{j, Z^{\prime}}^{\prime}\left(Z^{\prime}, \zeta^{\prime}\right) \in \mathbb{C} \llbracket Z^{\prime}, \zeta^{\prime} \rrbracket^{N^{\prime}}$, and $\rho_{j, Z^{\prime}}^{\prime}(H(Z), \bar{H}(\zeta)) \in \mathbb{C} \llbracket Z, \zeta \rrbracket^{N^{\prime}}$. We define an ascending chain of submodules $E_{k} \subset \mathbb{C} \llbracket Z, \zeta \rrbracket^{N^{\prime}}$ by

$$
E_{k}=\operatorname{span}_{\mathbb{C} \llbracket Z, \zeta \rrbracket}\left\{L_{1} \cdots L_{r} \rho_{j, Z^{\prime}}^{\prime}(H(Z), \bar{H}(\zeta)):\right.
$$

$L_{1}, \ldots, L_{r}$ formal CR-vector fields tangent to $\left.M, 0 \leq r \leq k, 1 \leq j \leq d^{\prime}\right\}$. 
The chain $E_{k}(0)$ of subspaces of $\mathbb{C}^{N^{\prime}}$ will stabilize, say at the index $k_{0}$, that is, $E_{k}(0)=E_{k_{0}}(0)$ for $k \geq k_{0}$, and $E_{k_{0}-1}(0) \neq E_{k_{0}}(0)$. So will the chain of submodules $E_{k} \subset \mathbb{C} \llbracket Z, \zeta \rrbracket^{N^{\prime}}$, since $\mathbb{C} \llbracket Z, \zeta \rrbracket^{N^{\prime}}$ as a free module over a Noetherian ring is Noetherian itself; say $E=\cup_{k} E_{k}$, and there is some $k_{0}^{\prime} \geq k_{0}$ such that $E_{k}=E$ for $k \geq k_{0}^{\prime}$.

Definition 13. With $E_{k_{0}}$ defined above, let $s=N^{\prime}-\operatorname{dim}_{\mathbb{C}} E_{k_{0}}(0)$. We then say that $H$ has formal degeneracy $\left(k_{0}, s\right.$ ) (at 0 ) or shortly, that $H$ is $\left(k_{0}, s\right)$-degenerate (at 0$)$. If $E$ is of constant rank $N^{\prime}-s$ over $I$, we say that $H$ has constant degeneracy $s$ and that $H$ is constantly $\left(k_{0}, s\right)$-degenerate. Furthermore, if $s=0$, we say that $H$ is $k_{0}$-nondegenerate. Since in that case, $E=\mathbb{C} \llbracket Z, \zeta \rrbracket^{N^{\prime}}, H$ has automatically constant degeneracy 0 .

Note that if $H$ is of constant degeneracy, then the submodule $E_{k_{0}}$ will actually generate $E$ up to vectors whose components are in $I$ (this follows from matrix manipulations, see Lemma 12). However, in general, we do not know whether $k_{0}=k_{0}^{\prime}$. Clearly, the degeneracy $s$ fulfills the inequality $0 \leq s \leq N^{\prime}-d^{\prime}$. Without further restrictions on $M, M^{\prime}$ and $H$, this is the best we can hope for, as the example of a Levi-flat submanifold as the target shows: If $M^{\prime}$ is defined by the equations $\operatorname{Im} w_{1}^{\prime}=\cdots=\operatorname{Im} w_{d}^{\prime}=0$, where $Z^{\prime}=\left(z^{\prime}, w^{\prime}\right)=\left(z_{1}^{\prime}, \ldots, z_{n}^{\prime}, w_{1}^{\prime}, \ldots, w_{d}^{\prime}\right)$ are coordinates in $\mathbb{C}^{N^{\prime}}$, then every map $H$ which maps into this manifold has constant degeneracy $N^{\prime}-d^{\prime}$.

We will now show that Definition 13 is actually independent of choices of formal coordinates and generators. First consider a different set of generators $\widetilde{\rho}^{\prime}=\left(\widetilde{\rho}_{1}^{\prime}, \ldots, \widetilde{\rho}_{d^{\prime}}^{\prime}\right)$. Then there is an invertible $d^{\prime} \times d^{\prime}$ matrix $A=\left(a_{j k}\right)$ of formal power series in $\mathbb{C} \llbracket Z, \zeta \rrbracket$ such that $\widetilde{\rho}^{\prime}\left(Z^{\prime}, \zeta^{\prime}\right)=A\left(Z^{\prime}, \zeta^{\prime}\right) \rho\left(Z^{\prime}, \zeta^{\prime}\right)$. Taking the complex gradient, we obtain

$$
\widetilde{\rho}_{j, Z^{\prime}}=\sum_{k=1}^{d^{\prime}} \rho_{k}^{\prime} a_{j k, Z^{\prime}}+\sum_{k=1}^{d^{\prime}} a_{j k} \rho_{k, Z^{\prime}}^{\prime}, \quad 1 \leq j \leq d^{\prime} .
$$

Now the first sum in (19) is a vector whose entries are elements of $I^{\prime}$. We write $\phi_{j}(Z, \zeta)=\rho_{j, Z^{\prime}}^{\prime}(H(Z), \bar{H}(\zeta)), \phi=\left(\phi_{1}, \ldots, \phi_{d^{\prime}}\right)$, use the same notation for $\widetilde{\rho}_{Z^{\prime}}^{\prime}$, and set $B(Z, \zeta)=A(H(Z), \bar{H}(\zeta))$; note that $B$ is an invertible $d^{\prime} \times d^{\prime}$ matrix of formal power series in $\mathbb{C} \llbracket Z, \zeta \rrbracket$. Then pulling (19) to $M$ via $H$, we see that

$$
\widetilde{\phi}_{j}=v_{j}+B \phi, \quad 1 \leq j \leq d^{\prime}
$$

where $v_{j}$ is a vector in $I \mathbb{C} \llbracket Z, \zeta \rrbracket^{N^{\prime}}$. (20) implies that $\widetilde{E}_{k}=E_{k}$ modulo $I \mathbb{C} \llbracket Z, \zeta \rrbracket^{N^{\prime}}$ for each $k$, which implies that $\operatorname{dim} \widetilde{E}_{k}(0)=\operatorname{dim} E_{k}(0)=e$ and that $\widetilde{E}_{k}$ is of constant rank e if and only if $E_{k}$ is. This shows that the choice of defining function does not matter. 
Note that Definition 13 is independent of the choice of formal holomorphic coordinates in $\mathbb{C}^{N}$; that follows easily from the fact that such a biholomorphic change of coordinates $F$ pushes formal CR-vector fields tangent to $M$ to formal CR-vector fields tangent to $F(M)$. The independence from choice of formal holomorphic coordinates in $\mathbb{C}^{N^{\prime}}$ is proved in the next Lemma.

Lemma 14. Suppose $M \subset \mathbb{C}^{N}, M^{\prime} \subset \mathbb{C}^{N^{\prime}}$ are generic formal submanifolds of $\mathbb{C}^{N}$ and $\mathbb{C}^{N^{\prime}}$, respectively, and that $H: M \rightarrow M^{\prime}$ is a formal holomorphic map. Fix a set of generators of $I^{\prime} \subset \mathbb{C} \llbracket Z, \zeta \rrbracket$, and let $\widetilde{Z}^{\prime}=F\left(Z^{\prime}\right)$ be a formal holomorphic change of coordinates in $\mathbb{C}^{N^{\prime}}$. Fix a set of generators of the ideal $\widetilde{I}^{\prime}$ representing $M^{\prime}$ in the $\widetilde{Z}^{\prime}$-variables. Denote the space defined by (18) in the $\widetilde{Z}^{\prime}$-variables by $\widetilde{E}_{k}$, then

$$
\widetilde{E}_{k}\left(\frac{\partial F}{\partial Z^{\prime}}(H(Z))\right)=E_{k}
$$

modulo $I \mathbb{C} \llbracket Z, \zeta \rrbracket^{N^{\prime}}$.

Proof. Let $\widetilde{\rho}$ be the fixed generators for the ideal $\widetilde{I}^{\prime}$ representing $M^{\prime}$ in the coordinates $\widetilde{Z}^{\prime}$. Then we can choose $\rho^{\prime}=F^{\sharp} \widetilde{\rho}^{\prime}$ as generators for $I^{\prime} \subset \mathbb{C} \llbracket Z, \zeta \rrbracket$. We now take the complex gradient, and use the chain rule to obtain

$$
\rho_{j, Z^{\prime}}^{\prime}\left(Z^{\prime}, \zeta^{\prime}\right)=\left(\widetilde{\rho}_{j}\left(F\left(Z^{\prime}\right), \bar{F}\left(\zeta^{\prime}\right)\right)\right)_{Z^{\prime}}=\widetilde{\rho}_{j, \widetilde{Z}^{\prime}}\left(F\left(Z^{\prime}\right), \bar{F}\left(\zeta^{\prime}\right)\right) \frac{\partial F}{\partial Z^{\prime}}\left(Z^{\prime}\right) .
$$

Pulling (22) to $M$ and applying CR-vector fields tangent to $M$, we obtain (21), since all $(0,1)$-vector fields annihilate the entries of the matrix $\frac{\partial F}{\partial Z^{\prime}}(H(Z))$. We have already shown above that if we choose different generators for $I^{\prime}$ in the same variables, the spaces $E_{k}$ are equal modulo $I \mathbb{C} \llbracket Z, \zeta \rrbracket^{N^{\prime}}$.

Now the transformation $v=\left(v_{1}, \ldots, v_{N^{\prime}}\right) \mapsto v A$, where $A$ is an invertible $N^{\prime} \times N^{\prime}$ matrix of formal power series in $(Z, \zeta)$, is a module isomorphism of $\mathbb{C} \llbracket Z, \zeta \rrbracket^{N^{\prime}}$ which maps $I \mathbb{C} \llbracket Z, \zeta \rrbracket^{N^{\prime}}$ into itself. So Definition 13 is in fact independent of all the choices made there. The next Lemma, the proof of which we leave to the reader, gives a means of actually computing with Definition 13.

Lemma 15. Suppose $M \subset \mathbb{C}^{N}, M^{\prime} \subset \mathbb{C}^{N^{\prime}}$ are generic formal submanifolds of $\mathbb{C}^{N}$ and $\mathbb{C}^{N^{\prime}}$, respectively, and that $H: M \rightarrow M^{\prime}$ is a formal holomorphic map. Let $L_{1}, \ldots, L_{n}$ be a basis of the CR-vector fields tangent to $M$ (that is, a set of generators of $\mathcal{D}_{M}^{0,1} / I_{\mathcal{D}}^{0,1}$, see 2.1.1). For a multi-index $\alpha \in \mathbb{N}^{n}$, let $L^{\alpha}=L_{1}^{\alpha_{1}} \cdots L_{n}^{\alpha_{n}}$. Furthermore, let generators $\rho^{\prime}=\left(\rho_{1}^{\prime}, \ldots \rho_{d^{\prime}}^{\prime}\right)$ of $I^{\prime}$ be chosen. Define the submodules

$$
F_{k}=\operatorname{span}_{\mathbb{C} \llbracket Z, \zeta \rrbracket}\left\{L^{\alpha} \rho_{j, Z^{\prime}}^{\prime}(H(Z), \bar{H}(\zeta)):|\alpha| \leq k, 1 \leq j \leq d^{\prime}\right\}
$$


of $\mathbb{C} \llbracket Z, \zeta \rrbracket^{N^{\prime}}$. Let $E_{k}$ be defined by (18). Then $E_{k}(0)=F_{k}(0)$, and $E_{k}$ is of constant rank over $I$ if and only if $F_{k}$ is; hence, in order to determine the degeneracy of $H$, it suffices to consider the $F_{k}$.

We now want to give a different characterization of the degeneracy $s$ in the case of constant degeneracy. For that, we will formulate the conditions of Definition 13 in terms of formal normal coordinates (see 2.1.2). So $I^{\prime}$ is generated by the functions $\rho_{j}^{\prime}=w_{j}^{\prime}-Q_{j}^{\prime}\left(z^{\prime}, \chi^{\prime}, \tau^{\prime}\right), j=1, \ldots, d^{\prime}$, and we will write $H=(f, g)=\left(f_{1}, \ldots, f_{n^{\prime}}, g_{1}, \ldots, g_{d^{\prime}}\right)$ for $H$ in these normal coordinates. The complex gradient is easily computed to be

$$
\rho_{j, Z^{\prime}}^{\prime}=\left(-Q_{j, z_{1}^{\prime}}^{\prime}, \ldots,-Q_{j, z_{n^{\prime}}^{\prime}}^{\prime}, e_{j}\right), \quad 1 \leq j \leq d^{\prime},
$$

where $e_{j}$ is the $j$ th unit vector in $\mathbb{C}^{d^{\prime}}$. In particular, the last $d^{\prime}$ entries of any CR-derivative of length bigger than 0 of any $\rho_{j, Z^{\prime}}^{\prime}(H(Z), \bar{H}(\zeta))$ will be 0 . We will write $Q_{j, z_{k}^{\prime}}^{\prime}(f(z, w), \bar{f}(\chi, \tau), \bar{g}(\chi, \tau))=\phi_{j}^{k}(Z, \zeta)$. So $H$ is of constant degeneracy $s$ if and only if (after possibly reordering the $z^{\prime}$ variables) there exist $t=n^{\prime}-s$ multi-indices $\alpha^{1}, \ldots, \alpha^{t} \in \mathbb{N}^{n}$ and integers $l_{1}, \ldots, l_{t}, 1 \leq l_{j} \leq d^{\prime}$, such that the vectors $\left(L^{\alpha^{j}} \phi_{l_{j}}^{1}, \ldots, L^{\alpha^{j}} \phi_{l_{j}}^{t}\right), 1 \leq j \leq t$, evaluated at 0 form a basis of $\mathbb{C}^{t}$, and for all multi-indices $\beta \in \mathbb{N}^{n}$, all $k$, $t+1 \leq k \leq n^{\prime}$, and all $l, 1 \leq l \leq d^{\prime}$, the determinant

$$
\left|\begin{array}{cccc}
L^{\alpha^{1}} \phi_{l_{1}}^{1} & \ldots & L^{\alpha^{1}} \phi_{l_{1}}^{t} & L^{\alpha^{1}} \phi_{l_{1}}^{k} \\
\vdots & \ddots & \vdots & \vdots \\
L^{\alpha^{t}} \phi_{l_{t}}^{1} & \ldots & L^{\alpha^{t}} \phi_{l_{t}}^{t} & L^{\alpha^{t}} \phi_{l_{t}}^{k} \\
L^{\beta} \phi_{l}^{1} & \ldots & L^{\beta} \phi_{l}^{t} & L^{\beta} \phi_{l}^{k}
\end{array}\right| \in I .
$$

More specifically, if $H$ is constantly $\left(k_{0}, s\right)$-degenerate, one of the $\alpha_{t}$ must have length $k_{0}$. We can use this to formulate the following technical result:

Lemma 16. Assume that normal coordinates $(z, w)$ and $\left(z^{\prime}, w^{\prime}\right)$ have been chosen for $M$ and $M^{\prime}$, respectively, that $L_{1}, \ldots, L_{n}$ is a basis of the (formal) CR-vector fields tangent to $M$ and that $w_{j}^{\prime}-Q_{j}^{\prime}\left(z^{\prime}, \chi^{\prime}, \tau^{\prime}\right)$ are generators of $I^{\prime}$ as in 2.1.2. Let $H: M \rightarrow M^{\prime}$ be of constant degeneracy $s$, let $t=n^{\prime}-s$, and write $H^{\sharp} Q_{j, z_{k}^{\prime}}^{\prime}=\phi_{j}^{k}$. We can choose $t$ multi-indices $\alpha^{1}, \ldots \alpha^{t}$, and integers $l_{1}, \ldots, l_{t}, 1 \leq l_{j} \leq d^{\prime}$, such that (after possibly reordering the $z^{\prime}$ variables) the vectors $\left(L^{\alpha^{j}} \phi_{l_{j}}^{1}, \ldots, L^{\alpha^{j}} \phi_{l_{j}}^{t}\right), 1 \leq j \leq t$, evaluated at 0 , form a basis of $\mathbb{C}^{t}$. Then

$$
\Delta \phi_{l}^{k}-\sum_{m=1}^{t} \Delta_{m k} \phi_{l}^{m} \in I, \quad 1 \leq l \leq d^{\prime}, \quad t+1 \leq k \leq n^{\prime},
$$


where

$$
\Delta(z, w)=\left|\begin{array}{ccc}
L^{\alpha^{1}} \phi_{l_{1}}^{1} & \cdots & L^{\alpha^{1}} \phi_{l_{1}}^{t} \\
\vdots & \ddots & \vdots \\
L^{\alpha^{t}} \phi_{l_{t}}^{1} & \cdots & L^{\alpha^{t}} \phi_{l_{t}}^{t}
\end{array}\right|(z, w, 0, w), \quad \Delta(0) \neq 0,
$$

and

$$
\begin{aligned}
& \Delta_{m k}(z, w) \\
& =(-1)^{t+m}\left|\begin{array}{cccccc}
L^{\alpha^{1}} \phi_{l_{1}}^{1} & \ldots & \widehat{L^{\alpha^{1}} \phi_{l_{1}}^{m}} & \ldots & L^{\alpha^{1}} \phi_{l_{1}}^{t} & L^{\alpha^{1}}=\phi_{l_{1}}^{k} \\
\vdots & & \vdots & & \vdots & \vdots \\
L^{\alpha^{t}} \phi_{l_{t}}^{1} & \ldots & \widehat{L^{\alpha^{t}} \phi_{l_{t}}^{m}} & \ldots & L^{\alpha^{1}} \phi_{l_{1}}^{t} & L^{\alpha^{t}} \phi_{l_{t}}^{k}
\end{array}\right|(z, w, 0, w),
\end{aligned}
$$

and where the ${ }^{-}$means that this column has been dropped. More specifically, if $H$ is constantly $\left(k_{0}, s\right)$-degenerate, then the $\alpha^{j}$ can be chosen to fulfill $1 \leq$ $\left|\alpha^{j}\right| \leq k_{0}$, and the same choice of $\alpha^{j}$ is possible for every map $H^{\prime}: M \rightarrow M^{\prime}$ (of constant degeneracy) agreeing with $H$ up to order $k_{0}$.

Proof. We will be using the parametrization of $M$ as in 2.1.2. Note that by (7), for a formal series $\phi(z, w, \chi, \tau) \in I, \phi(z, w, 0, w)=0$. We use this in (25). Developing the resulting determinant along the last row, we see that for every $\beta \in \mathbb{N}^{n}$, for every $l, 1 \leq l \leq d^{\prime}$, and for every $k, t+1 \leq k \leq n^{\prime}$

$$
\Delta(z, w) L^{\beta} \phi_{l}^{k}(z, w, 0, w)-\sum_{m=1}^{t} \Delta_{m k}(z, w) L^{\beta} \phi_{l}^{m}(z, w, 0, w)=0,
$$

where $\Delta$ and $\Delta_{m k}$ are defined by (27) and (28), respectively. Recalling (12) we conclude that

$$
\Delta(z, w) \phi_{l}^{k}(z, w, \chi, \bar{Q}(\chi, z, w))-\sum_{m=1}^{t} \Delta_{m k}(z, w) \phi_{l}^{m}(z, w, \chi, \bar{Q}(\chi, z, w))=0 .
$$

This immediately implies (26). The last statement follows from the construction.

We are now going to characterize the degeneracy $s$ of a mapping of constant degeneracy in terms of certain formal holomorphic vector fields. These results generalize some results about holomorphic nondegeneracy (defined in $[\mathbf{1 5}]$ ) which can be found in e.g., [5].

Definition 17. Let $M \subset \mathbb{C}^{N}, M^{\prime} \subset \mathbb{C}^{N^{\prime}}$ be formal submanifolds of $\mathbb{C}^{N}$ and $\mathbb{C}^{N^{\prime}}$, respectively, $H: M \rightarrow M^{\prime}$ a formal holomorphic map. A formal 
holomorphic vector field $X$ in $\mathbb{C}^{N^{\prime}}$ tangent to $M^{\prime}$ along $H(M)$ is an operator of the form

$$
X=\sum_{j=1}^{N^{\prime}} a_{j}(Z) \frac{\partial}{\partial Z_{j}^{\prime}},
$$

where $a_{j} \in \mathbb{C} \llbracket Z \rrbracket$ (called the coefficients of $X$ ), with the property that for every $\phi \in I^{\prime}, \sum_{j=1}^{N^{\prime}} a_{j}(Z) \phi_{Z_{j}^{\prime}}(H(Z), \bar{H}(\zeta)) \in I$. We say that a set $\left\{X_{1}, \ldots, X_{l}\right\}$ of such formal holomorphic vector fields is linearly independent if their coefficients evaluated at 0 form a linearly independent set of vectors in $\mathbb{C}^{N^{\prime}}$.

If $H$ is an immersion, one can associate to $X$ as in Definition 17 a formal holomorphic vector field in $\mathbb{C}^{N^{\prime}}$ in the following way. $H(M)$ can be regarded as a formal submanifold (not generic) of $\mathbb{C}^{N^{\prime}}$ by taking as its ideal $\left(H^{\sharp}\right)^{-1}(I) \supset I^{\prime}$. Since $H$ is immersive, $H$ has a right inverse $G$, so $H^{\sharp}$ has a left inverse $G^{\sharp}$. Hence, an expression of the form $X=\sum_{j=1}^{N^{\prime}} a_{j}(Z) \frac{\partial}{\partial Z_{j}^{\prime}}$ gives rise to a formal holomorphic vector field $X^{\prime}=\sum_{j=1}^{N^{\prime}} G^{\sharp} a_{j}\left(Z^{\prime}\right) \frac{\partial}{\partial Z_{j}^{\prime}}$ in $\mathbb{C}^{N^{\prime}}$, and $X$ is tangent to $M^{\prime}$ along $H(M)$ if and only if $H^{\sharp}\left(X^{\prime} f\right) \in I$ for all $f \in I^{\prime}$.

Also note that it is enough to check the condition in Definition 17 on a set of generators of $I$, and that the space of all holomorphic vector fields tangent to $M^{\prime}$ along $H(M)$ can be identified with a submodule of the free module $\mathbb{C} \llbracket Z \rrbracket^{N^{\prime}}$.

Example 2. Consider the standard linear injection of the boundary of the $N$-ball $\left|Z_{1}\right|^{2}+\cdots+\left|Z_{N}\right|^{2}=1$ into the boundary of the $N^{\prime}$-ball $\left|Z_{1}\right|^{2}+$ $\cdots+\left|Z_{N^{\prime}}\right|^{2}=1,\left(Z_{1}, \ldots, Z_{N}\right) \mapsto\left(Z_{1}, \ldots, Z_{N}, 0, \ldots, 0\right)$. This map is of constant degeneracy $N^{\prime}-N$ everywhere and there are $N^{\prime}-N$ linearly independent holomorphic vector fields tangent to $M^{\prime}$ along $H(M)$ given by $\frac{\partial}{\partial Z_{N+1}^{\prime}}, \ldots, \frac{\partial}{\partial Z_{N^{\prime}}^{\prime}}$.

The situation in Example 2 is typical in the following sense:

Proposition 18. Assume that $M \subset \mathbb{C}^{N}, M^{\prime} \subset \mathbb{C}^{N^{\prime}}$ are generic formal submanifolds of $\mathbb{C}^{N}$ and $\mathbb{C}^{N^{\prime}}$, respectively, and $H: M \rightarrow M^{\prime}$ is of constant degeneracy s. Then

$$
s=\operatorname{dim}_{\mathbb{C}}\{X(0): X \text { formal holomorphic vector field }
$$

$$
\text { tangent to } \left.M^{\prime} \text { along } H(M)\right\} \text {. }
$$

Proof. Let $\widetilde{s}$ denote the dimension of the space on the right hand side of (32). From Lemma 16 we see that there are at least $s$ linearly independent holomorphic vector fields along $H$ tangent to $M^{\prime}$ along $H(M)$, so that $s \leq \widetilde{s}$. In fact, choosing local holomorphic coordinates $(z, w)$ for $M,\left(z^{\prime}, w^{\prime}\right)$ for $M^{\prime}$ 
and a defining function $Q^{\prime}=\left(Q_{1}^{\prime}, \ldots, Q_{d^{\prime}}^{\prime}\right)$ for $M^{\prime}$ as in Lemma 16, we have that the formal holomorphic vector fields

$$
X_{k}=\frac{\partial}{\partial z_{k}^{\prime}}-\sum_{m=1}^{t} a_{m, k}(z, w) \frac{\partial}{\partial z_{m}^{\prime}}, \quad k=t+1, \ldots, n^{\prime},
$$

are tangent to $M^{\prime}$ along $H(M)$ by $(26)$ where $a_{m, k}(z, w)=\Delta_{m k}(z, w) / \Delta(z, w)$ and $\Delta_{m k}$ and $\Delta$ are defined by (28) and (27), respectively.

On the other hand, assume that $\left\{X_{1}, \ldots X_{\widetilde{s}}\right\}$ are linearly independent holomorphic vector fields tangent to $M^{\prime}$ along $H(M)$; say $X_{k}=$ $\sum_{j=1}^{N^{\prime}} a_{j}^{k}(Z) \frac{\partial}{\partial Z_{j}^{\prime}}$, and let $\rho^{\prime}=\left(\rho_{1}^{\prime}, \ldots, \rho_{d^{\prime}}^{\prime}\right)$ be a set of generators for $I^{\prime}$. We have that

$$
\sum_{j=1}^{N^{\prime}} a_{j}^{k}(Z) \rho_{l, Z_{j}^{\prime}}^{\prime}(H(Z), \bar{H}(\zeta)) \in I, \quad 1 \leq l \leq d^{\prime} .
$$

Applying CR-vector fields $L_{1}, \ldots, L_{r}$ tangent to $M$ to (34), we see that

$$
\sum_{j=1}^{N^{\prime}} a_{j}^{k}(Z) L_{1} \cdots L_{r} \rho_{l, Z_{j}^{\prime}}^{\prime}(H(Z), \bar{H}(\zeta)) \in I, \quad 1 \leq l \leq d^{\prime} .
$$

Evaluating (35) at 0 , we conclude that $\operatorname{dim}_{\mathbb{C}} E_{k}(0) \leq N^{\prime}-\widetilde{s}$ for all $k$. Hence, $\widetilde{s} \leq s$, and the proof is complete.

Note that the second part of the proof of Proposition 18 shows that if we denote the dimension of the space on the right hand side of (32) by $\widetilde{s}$, then the degeneracy $s$ of a formal map $H$ always satisfies $\widetilde{s} \leq s$, whether the degeneracy is constant or not.

We now want to relate our notion of nondegeneracy of a map with the notion of finite nondegeneracy of manifolds. In particular, we give a bound on the degeneracy for a certain class of maps between finitely nondegenerate manifolds.

2.4. Finitely nondegenerate manifolds. The notion of finite nondegeneracy was introduced for hypersurfaces in [6], and has been used extensively in the study of mapping problems. We say that a generic submanifold is finitely nondegenerate (or, more specifically, $\ell_{0}$-nondegenerate) if its identity map is $\ell_{0}$-nondegenerate in the sense of Definition 13 . For the original definition, see e.g., [5], Chapter IX. By the chain rule we see that if there is a $k_{0}$-nondegenerate map into some generic formal submanifold $M^{\prime} \subset \mathbb{C}^{N^{\prime}}$, then $M^{\prime}$ is $\ell_{0}$-nondegenerate for some $\ell_{0} \leq k_{0}$. In fact, we also see that every formal biholomorphism between generic formal submanifolds $M \subset \mathbb{C}^{N}$ and $M^{\prime} \subset \mathbb{C}^{N}$ of the same codimension which are $\ell_{0}$-nondegenerate is in fact $\ell_{0}$-nondegenerate. 
In order to use finite nondegeneracy of submanifolds to put bounds on the degeneracy, we need the mapping to fulfill another crucial property, which we will introduce next.

Definition 19. Let $M \subset \mathbb{C}^{N}, M^{\prime} \subset \mathbb{C}^{N^{\prime}}$ be formal generic submanifolds in $\mathbb{C}^{N}$ and $\mathbb{C}^{N^{\prime}}$, respectively, $H: M \rightarrow M^{\prime}$ a formal holomorphic map. We say that $H$ is transversal if for one (and hence every) set of generators $\rho^{\prime}=\left(\rho_{1}^{\prime}, \ldots, \rho_{d^{\prime}}^{\prime}\right)$ of $I^{\prime}, H^{\sharp} \rho^{\prime}$ generates $I$.

Note that in particular, if $H: M \rightarrow M^{\prime}$ is transversal, then $d \leq d^{\prime}$. Transversality is most easily expressed after choosing normal coordinates $(z, w) \in \mathbb{C}^{n} \times \mathbb{C}^{d}$ for $M$ and $\left(z^{\prime}, w^{\prime}\right) \in \mathbb{C}^{n^{\prime}} \times \mathbb{C}^{d^{\prime}}$ for $M^{\prime}$. Write $H=(f, g)$ in these coordinates. Then $H$ is transversal if and only if the matrix $\frac{\partial g}{\partial w}(0)$ has maximal rank $d$.

Lemma 20. Let $M \subset \mathbb{C}^{N}, M^{\prime} \subset \mathbb{C}^{N^{\prime}}$ be formal generic submanifolds of $\mathbb{C}^{N}$ and $\mathbb{C}^{N^{\prime}}$, respectively, with $M$ being $\ell_{0}$-nondegenerate, and $H: M \rightarrow M^{\prime}$ a transversal mapping. Then the degeneracy s of $H$ fulfills $0 \leq s \leq N^{\prime}-N$.

Proof. Assume that $\rho^{\prime}=\left(\rho_{1}^{\prime}, \ldots, \rho_{d^{\prime}}^{\prime}\right)$ generates $I^{\prime}$. Without loss of generality, assume that $\left(H^{\sharp} \rho_{1}^{\prime}, \ldots, H^{\sharp} \rho_{d}^{\prime}\right)$ generate $I$. Now using the chain rule it follows that

$$
\left(\rho_{j}^{\prime}(H(Z), \bar{H}(\zeta))\right)_{Z}=\rho_{j, Z^{\prime}}^{\prime}(H(Z), \bar{H}(\zeta)) \frac{\partial H}{\partial Z}(Z), \quad j=1, \ldots, d .
$$

Applying CR-vector fields $L_{1}, \ldots, L_{r}$ tangent to $M$ to (36), we see that

$$
\begin{aligned}
& L_{1} \cdots L_{r}\left(\rho_{j}^{\prime}(H(Z), \bar{H}(\zeta))\right)_{Z}= \\
& \quad\left(L_{1} \cdots L_{r} \rho_{j, Z^{\prime}}^{\prime}(H(Z), \bar{H}(\zeta))\right) \frac{\partial H}{\partial Z}(Z), \quad j=1, \ldots, d .
\end{aligned}
$$

By hypothesis, if evaluated at 0 , the dimension of the space spanned by the vectors on the right hand side of (37) is $N$. On the other hand, the span of the vectors $L_{1} \cdots L_{r} \rho_{j, Z^{\prime}}^{\prime}(H(Z), \bar{H}(\zeta))$ evaluated at 0 has dimension $N^{\prime}-s$, where $s$ is the degeneracy of $H$. Hence, (37) implies that $N \leq N^{\prime}-s$, which is the inequality claimed.

2.5. Real-analytic and smooth submanifolds. We now want to apply the theory developed above to smooth submanifolds of $\mathbb{C}^{N}$ and $\mathbb{C}^{N^{\prime}}$. First, let $M \subset \mathbb{C}^{N}$ and $M^{\prime} \subset \mathbb{C}^{N^{\prime}}$ be generic $C^{\infty}$-submanifolds of $\mathbb{C}^{N}$ and $\mathbb{C}^{N^{\prime}}$ of codimension $d$ and $d^{\prime}$, respectively. Assume that $p_{0} \in M, p_{0}^{\prime} \in M^{\prime}$, and $H$ is a holomorphic mapping (or, more generally, a $C^{\infty}$-CR-mapping) defined in a neighbourhood $U$ of $p_{0}$, with $H\left(p_{0}\right)=p_{0}^{\prime}$ and $H(U \cap M) \subset M^{\prime}$. We write $\mathcal{V}(M)$ for the CR-bundle of $M$, i.e., the bundle with $\mathcal{V}(M)_{p}=$ $\mathbb{C} T_{p} M \cap \mathbb{C} T_{p}^{(0,1)} \mathbb{C}^{N}$. 
To $M$ and $M^{\prime}$ we associate formal submanifolds of $\mathbb{C}^{N}$ and $\mathbb{C}^{N^{\prime}}$, respectively, by choosing holomorphic coordinates $Z$ and $Z^{\prime}$ in $\mathbb{C}^{N}$ and $\mathbb{C}^{N^{\prime}}$, respectively, in which $p_{0}=0$ and $p_{0}^{\prime}=0$ and assigning them the ideals $I \subset \mathbb{C} \llbracket Z, \zeta \rrbracket$ and $I^{\prime} \subset \mathbb{C} \llbracket Z^{\prime}, \zeta^{\prime} \rrbracket$ which are generated by the Taylor series of their defining functions. $H$ corresponds to a formal holomorphic map-by its Taylor expansion, if it is holomorphic, and by its formal holomorphic power series (see [5], §1.7.) if it is $C^{\infty}-\mathrm{CR}$. Also, a local basis $L_{1}, \ldots L_{n}$ of the CR-vector fields tangent to $M$ gives rise (by taking the Taylor expansion of the coefficients) to a basis for the formal CR-vector fields tangent to the formal manifold M.

Abusing notation, we shall always use the same letters to denote the formal object associated to a concrete object; this will cause no confusion, since the operations done on them clearly distinguish the two classes.

Choose defining functions $\rho^{\prime}=\left(\rho_{1}^{\prime}, \ldots, \rho_{d^{\prime}}^{\prime}\right)$ for $M^{\prime}$ and a local basis $L_{1}, \ldots, L_{n}$ for $C^{\infty}(M, \mathcal{V}(M))$. As above, for a multi-index $\alpha \in \mathbb{N}^{n}$, we write $L^{\alpha}=L_{1}^{\alpha_{1}} \ldots L_{n}^{\alpha_{n}}$. After possibly shrinking $U$, we can define the vector subspaces

$$
E_{k}^{\prime}(p)=\operatorname{span}_{\mathbb{C}}\left\{\left.L^{\alpha} \rho_{j, Z^{\prime}}^{\prime}(H(Z), \overline{H(Z)})\right|_{Z=p}:|\alpha| \leq k, 1 \leq j \leq d^{\prime}\right\} \subset \mathbb{C}^{N^{\prime}}
$$

for $p \in U$. Let $s(p)=N^{\prime}-\max _{k} \operatorname{dim}_{\mathbb{C}} E_{k}^{\prime}(p)$. We can then say that $H$ is of degeneracy $s(0)$ at 0 , and that $H$ is of constant degeneracy $s$ at 0 if $s(p)$ is constant on a neighbourhood of 0 in $M$. By taking $k_{0}$ to be the least integer for which $E_{k}^{\prime}(0)=E_{k_{0}}^{\prime}(0)$ for $k \geq k_{0}$, we can also define the finer invariant of $\left(k_{0}, s\right)$-degeneracy, like in Definition 13. Just as in the case of formal degeneracy, one sees that this definition is in fact independent of the choices made, and invariant under biholomorphic changes of coordinates in both $\mathbb{C}^{N}$ and $\mathbb{C}^{N^{\prime}}$.

Finally, as noted above, the notion of $k_{0}$-nondegeneracy makes sense for mappings which are a priori only assumed to be $C^{k_{0}}$. This was used in [12] to prove a reflection principle, and is used in the statement of Corollary 4.

In the case of real-analytic submanifolds, we can give generic bounds on both $k_{0}$ and $s$ (under some additional assumptions), which we want to do now.

Definition 21. Let $M$ and $M^{\prime}$ be connected, real-analytic, generic submanifolds of $\mathbb{C}^{N}$ and $\mathbb{C}^{N^{\prime}}$, respectively, and $H$ a holomorphic mapping defined on an open set $U \in \mathbb{C}^{N}$ containing $M$ with $H(M) \subset M^{\prime}$. For all $p \in M$, let $s(p)$ be the degeneracy of $H$ at $p$. The generic degeneracy $s(H)$ is defined as $s(H)=\min _{p \in M} s(p)$.

The following Lemma implies that the set of points where $H$ is of constant degeneracy $s(H)$ is an open, dense subset of $M$. 
Lemma 22. Let $M, M^{\prime}, H$ be as in Definition 21. The set $\{p \in M: s(p)>$ $s(H)\}$ is real-analytic.

Proof. After choosing local defining functions, the points where the degeneracy of $H$ is strictly bigger than $s(H)$ is given by the vanishing of determinants with real-analytic entries; see the arguments before (25).

The inequality $0 \leq s(H) \leq N^{\prime}-d^{\prime}$ holds trivially. The upper bound corresponding to Lemma 20 is sharper:

Lemma 23. Let $M, M^{\prime}$ and $H$ be as in Definition 21 and assume in addition that there exists a point $p_{0}^{\prime} \in H(M)$ at which $M^{\prime}$ is finitely nondegenerate and that $H$ is transversal. Then $0 \leq s(H) \leq N^{\prime}-N$.

The proof is immediate from Lemma 20 .

We are now going to derive a bound on $k_{0}$. Assume for simplicity that $0 \in M, 0 \in M^{\prime}$, and that $H(M) \subset M^{\prime}$ with $H(0)=0$. Also let normal coordinates $(z, w)$ for $M$ and $\left(z^{\prime}, w^{\prime}\right)$ for $M^{\prime}$ with corresponding real-analytic functions $Q: \mathbb{C}^{2 n+d} \rightarrow \mathbb{C}^{d}$ and $Q^{\prime}: \mathbb{C}^{2 n^{\prime}+d^{\prime}} \rightarrow \mathbb{C}^{d^{\prime}}$ (each defined and convergent in a neighbourhood of $0 \in \mathbb{C}^{2 n+d}$ and $\left.0 \in \mathbb{C}^{2 n^{\prime}+d^{\prime}}\right)$ be chosen. That is, both $Q$ and $Q^{\prime}$ fulfill (7), $M$ is given by $w=Q(z, \bar{z}, \bar{w})$ in a neighbourhood of 0 , and $M^{\prime}$ is given by $w^{\prime}=Q^{\prime}\left(z^{\prime}, \bar{z}^{\prime}, \bar{w}^{\prime}\right)$. As in 2.3 , we write $H=(f, g)$ and set $Q_{j, z_{k}^{\prime}}^{\prime}(f(z, w), \overline{f(z, w)}, \overline{g(z, w)})=\phi_{j}^{k}(z, w, \bar{z}, \bar{w})$. If $H$ is of constant degeneracy $s$ at 0 , say $H$ is $\left(s, k_{0}\right)$-degenerate at 0 , then after reordering we may assume that (writing $e=n^{\prime}-s$ ) the vector valued functions

$$
\phi_{j}=\left(\phi_{j}^{1}, \ldots, \phi_{j}^{e}, e_{j}\right), \quad 1 \leq j \leq d^{\prime},
$$

where $e_{j}$ is the $j$ th unit vector in $\mathbb{C}^{d^{\prime}}$, are real-analytic at $0 \in \mathbb{C}^{N}$; they are clearly linearly independent at 0 , and furthermore, if we choose the basis of CR-vector fields tangent to $M$

$$
L_{k}=\frac{\partial}{\partial \bar{z}_{k}}+\sum_{j=1}^{d} \bar{Q}_{j \bar{z}_{k}}(\bar{z}, z, w) \frac{\partial}{\partial \bar{w}_{j}}, \quad 1 \leq k \leq d,
$$

and let $L^{\alpha}=L_{1}^{\alpha_{1}} \cdots L_{n}^{\alpha_{n}}$, then the set $\left\{\left.L^{\alpha} \phi_{j}\right|_{0}: \alpha \in \mathbb{N}^{n}, 1 \leq j \leq d^{\prime}\right\}$ spans $\mathbb{C}^{N^{\prime}-s}$. Now we can complexify all of these statements. So we let $\mathcal{M}=\left\{(z, w, \chi, \tau) \in U \subset \mathbb{C}^{n} \times \mathbb{C}^{d} \times \mathbb{C}^{n} \times \mathbb{C}^{d}: \tau_{j}=\bar{Q}_{j}(\chi, z, w)\right\}$ where $\mathrm{U}$ is a neighbourhood of 0 on which $Q$ is convergent be the complexification of $M$; $\mathcal{M}$ is a holomorphic submanifold of codimension $d$ in $\mathbb{C}^{2 N}$. We also need the submanifold $\mathcal{M}_{0}$ of dimension $n$ defined by $\mathcal{M}_{0}=\left\{(\chi, \tau) \in \mathbb{C}^{n} \times \mathbb{C}^{d}: \tau_{j}=\right.$ $\left.\bar{Q}_{j}(\chi, 0,0)\right\}=\left\{(\chi, 0): \chi \in \mathbb{C}^{n}\right\}$. The complexifications of the $L_{k}$ are

$$
\mathcal{L}_{k}=\frac{\partial}{\partial \chi_{k}}+\sum_{j=1}^{d} \bar{Q}_{j, \chi_{k}}(\chi, z, w) \frac{\partial}{\partial \tau_{j}}, \quad 1 \leq k \leq d,
$$


and if we denote the complexification of $\phi_{j}$ again by $\phi_{j}$, then we have that the set $\left\{\left.\mathcal{L}^{\alpha} \phi_{j}\right|_{0}: \alpha \in \mathbb{N}^{n}, 1 \leq j \leq d^{\prime}\right\}$, spans $\mathbb{C}^{N^{\prime}-s}$. Now note that we can restrict our attention to $\mathcal{M}_{0}$ in this statement, since none of the $\mathcal{L}_{k}$ differentiates in $z$ or $w$; hence, we have that

$$
\operatorname{span}_{\mathbb{C}}\left\{\left.\frac{\partial^{|\alpha|}}{\partial \chi^{\alpha}} \phi_{j}(0,0, \chi, 0)\right|_{\chi=0}: \alpha \in \mathbb{N}^{n}, 1 \leq j \leq d^{\prime}\right\}=\mathbb{C}^{N^{\prime}-s} .
$$

We now apply e.g., Lemma 11.5.4. in [5] to conclude that generically, the derivatives of $\phi_{j}(0,0, \chi, 0)$ up to order $N^{\prime}-s-d^{\prime}$ span $\mathbb{C}^{N^{\prime}-s}$; which in turn implies that generically, $k_{0} \leq N^{\prime}-d^{\prime}-s$. We summarize:

Lemma 24. Let $M$ and $M^{\prime}$ be connected, real-analytic, generic submanifolds of $\mathbb{C}^{N}$ and $\mathbb{C}^{N^{\prime}}$, respectively, and $H$ a holomorphic mapping defined on an open set $U \in \mathbb{C}^{N}$ containing $M$ with $H(M) \subset M^{\prime}$. Then there exist numbers $s \leq N^{\prime}-d^{\prime}$ and $k_{0} \leq N^{\prime}-d^{\prime}-s$ such that outside some proper real analytic subvariety of $M, H$ is $\left(k_{0}, s\right)$-degenerate.

\section{Nondegenerate mappings.}

In this section we shall discuss nondegenerate mappings. We start with the "basic identity", and in the next subsection, prove Theorems 1, 2, and 5.

3.1. The basic identity. We write $K(t)=\left|\left\{\alpha \in \mathbb{N}^{N}:|\alpha| \leq t\right\}\right|$ for the number of all multi-indices of length less than $t$. For a multi-index $\alpha, \partial^{\alpha}$ denotes the operator $\frac{\partial^{|\alpha|}}{\partial Z^{\alpha}}$. The following proposition is our starting point.

Proposition 25 (Basic identity for nondegenerate maps). Let $M \subset \mathbb{C}^{N}$, $M^{\prime} \subset \mathbb{C}^{N^{\prime}}$ be generic formal submanifolds, $H: M \rightarrow M^{\prime}$ a formal holomorphic map which is $k_{0}$-nondegenerate. Then there exists a formal function $\Psi: \mathbb{C}^{N} \times \mathbb{C}^{N} \times \mathbb{C}^{K\left(k_{0}\right) N^{\prime}} \rightarrow \mathbb{C}^{N^{\prime}}$ (that is, if we write $W$ for the coordinates in $\left.\mathbb{C}^{K\left(k_{0}\right) N^{\prime}}, \Psi \in \mathbb{C} \llbracket Z, \zeta, W \rrbracket^{N^{\prime}}\right)$ with the property that

$$
H(Z)-\Psi\left(Z, \zeta,\left(\partial^{\beta} \bar{H}(\zeta)-\partial^{\beta} \bar{H}(0)\right)_{0 \leq|\beta| \leq k_{0}}\right) \in I^{N^{\prime}}=\underbrace{I \times \cdots \times I}_{N^{\prime} \text { times }} ;
$$

furthermore, $\Psi$ depends only on $M, M^{\prime}$, and on the values of $\partial^{\beta} H(0)$ for $\beta \leq\left|k_{0}\right|$, such that if $H^{\prime}: M \rightarrow M^{\prime}$ is another formal map with $\partial^{\beta} H(0)=$ $\partial^{\beta} H^{\prime}(0)$ for $|\beta| \leq k_{0}$, then (43) holds with $H^{\prime}$ in place of $H$. If $M$ and $M^{\prime}$ are real-analytic, $\Psi$ is convergent on a neighbourhood of the origin. If $M$ and $M^{\prime}$ are algebraic, so is $\Psi$.

Proof. Choose a basis $L_{1}, \ldots, L_{n}$ of the CR-vector fields tangent to $M$ and defining functions $\rho^{\prime}=\left(\rho_{1}^{\prime}, \ldots, \rho_{d^{\prime}}^{\prime}\right)$. By Lemma 15 , we can choose $N^{\prime}$ multiindices $\alpha^{1}, \ldots \alpha^{N^{\prime}}$ and integers $l^{1}, \ldots, l^{N^{\prime}}$ with $0 \leq\left|\alpha^{j}\right| \leq k_{0}, 1 \leq l^{j} \leq d^{\prime}$ for 
all $j=1, \ldots, N^{\prime}$ such that

$$
\operatorname{det}\left(\left.L^{\alpha^{j}} \rho_{l^{j}, Z_{k}^{\prime}}^{\prime}(H(Z), \bar{H}(\zeta))\right|_{0}\right)_{\substack{1 \leq j \leq N^{\prime} \\ 1 \leq k \leq N^{\prime}}} \neq 0 .
$$

We write $\Phi_{j}\left(Z, \zeta, H(Z), \bar{H}(\zeta),\left(\partial^{\beta} \bar{H}(\zeta)\right)_{1 \leq|\beta| \leq k_{0}}\right)=L^{\alpha^{j}} \rho_{l^{j}}^{\prime}(H(Z), \bar{H}(\zeta)) \in I$ for $1 \leq j \leq N^{\prime}$; using the chain rule, we see that $L^{\alpha} \Phi_{j} \in \mathbb{C} \llbracket Z, \zeta, Z^{\prime}, \zeta^{\prime} \rrbracket[W]$ where $W$ are variables in $\mathbb{C}^{\left(K\left(k_{0}\right)-1\right) N^{\prime}}$. We make a change of variables by replacing $W$ by $W+\partial^{\beta} \bar{H}(0)_{1 \leq|\beta| \leq k_{0}}$ and write again $\Phi_{j}$ in these new variables; hence, $\Phi_{j}\left(Z, \zeta, H(Z), \bar{H}(\zeta),\left(\partial^{\beta} \bar{H}(\zeta)-\partial^{\beta} \bar{H}(0)\right)_{1 \leq|\beta| \leq k_{0}}\right) \in I$, and $\Phi_{j} \in \mathbb{C} \llbracket Z, \zeta, Z^{\prime}, \zeta^{\prime} \rrbracket[W]$; also, $\Phi_{j}$ depends only on $M, M^{\prime}$, and on the values of $\partial^{\beta} H(0)$ for $|\beta| \leq k_{0}$.

Now consider the equations

$$
\Phi_{j}\left(Z, \zeta, Z^{\prime}, \zeta^{\prime}, W\right)=0, \quad 1 \leq j \leq N^{\prime} .
$$

We claim that this family of equations has a unique solution in $Z^{\prime}$. In fact, if we compute the Jacobian of (45) with respect to $Z^{\prime}$ at 0 , by the definition of $\Phi_{j}$ and using (44), we see that the Jacobian matrix $\left(\frac{\partial \Phi_{j}}{\partial Z_{k}^{\prime}}(0)\right)_{j, k}$ is nonsingular. It follows by the formal implicit function theorem that there exist $N^{\prime}$ unique formal power series $\Psi_{j} \in \mathbb{C} \llbracket Z, \zeta, \zeta^{\prime}, W \rrbracket, j=1, \ldots, N^{\prime}$, with the property that $\Phi_{j}\left(Z, \zeta, \Psi_{1}\left(Z, \zeta, \zeta^{\prime}, W\right), \ldots, \Psi_{N^{\prime}}\left(Z, \zeta, \zeta^{\prime}, W\right), \zeta^{\prime}, W\right)=0$.

We recall that $\Phi_{j}\left(Z, \zeta, H(Z), \bar{H}(\zeta),\left(\partial^{\beta} \bar{H}(\zeta)-\partial^{\beta} \bar{H}(0)\right)_{1 \leq|\beta| \leq k_{0}}\right) \in I$; if we replace $Z$ and $\zeta$ by a parametrization (as, for example, in 2.1.2) of $I$, say $Z(x)$ and $\zeta(x)$, we conclude that $\Phi_{j}\left(Z(x), \zeta(x), H(Z(x)),\left(\partial^{\beta} \bar{H}(\zeta(x))-\right.\right.$ $\left.\left.\partial^{\beta} \bar{H}(0)\right)_{1 \leq|\beta| \leq k_{0}}\right)=0$. It follows that $H_{j}(Z(x))=\Psi_{j}(Z(x), \zeta(x), \bar{H}(\zeta(x))$, $\left.\left(\partial^{\beta} \bar{H}(\zeta(x))-\partial^{\beta} \bar{H}(0)\right)_{1 \leq|\beta| \leq k_{0}}\right), 1 \leq j \leq N^{\prime}$. Passing back to the ring $\mathbb{C} \llbracket Z, \zeta \rrbracket$, we conclude that $\Psi=\left(\Psi_{1}, \ldots, \Psi_{N^{\prime}}\right)$ fulfills (43).

By construction, the map $\Phi$ depends only on $M, M^{\prime}$, and $\partial^{\beta} H(0), 0 \leq$ $|\beta| \leq k_{0}$. The same choice of $\alpha^{1}, \ldots, \alpha^{N^{\prime}}$ and $l^{1}, \ldots l^{N^{\prime}}$ works for every other map $H^{\prime}$ with $\partial^{\beta} H^{\prime}(0)=\partial^{\beta} H(0),|\beta| \leq k_{0}$. Finally, if $M$ and $M^{\prime}$ are real analytic or algebraic, we can choose the defining functions and the basis of CR-vector fields to be real-analytic (or algebraic, respectively) and the last two claims of Proposition 25 follow since those classes of maps are closed under application of the implicit function theorem.

We shall need some formal vector fields tangent to $M$, which will help us to exploit (43). Let $\rho=\left(\rho_{1}, \ldots, \rho_{d}\right)$ be a real-analytic defining function for $M$. After renumbering, we may assume that $\rho_{\hat{\zeta}}=\left(\frac{\partial \rho_{j}}{\partial \zeta_{k}}\right)_{1 \leq j, k \leq d}$ is invertible; set

$$
S_{j}=\frac{\partial}{\partial Z_{j}}-\rho_{Z_{j}}\left(\rho_{\hat{\zeta}}\right)^{-1} \frac{\partial}{\partial \hat{\zeta}}, \quad j=1, \ldots, N
$$


where $\frac{\partial}{\partial \hat{\zeta}}=\left(\frac{\partial}{\partial \zeta_{1}}, \ldots, \frac{\partial}{\partial \zeta_{d}}\right)^{t}$. Then $S_{j}$ is a (formal) vector field tangent to $M$, and its coefficients are convergent, if $M$ is assumed to be real-analytic, and algebraic functions if $M$ is assumed to be algebraic. If for $\alpha \in \mathbb{N}^{N}$ we write $S^{\alpha}=S_{1}^{\alpha_{1}} \cdots S_{N}^{\alpha_{N}}$, then for $H \in \mathbb{C} \llbracket Z \rrbracket, S^{\alpha} H=\partial^{\alpha} H$. Applying these vector fields repeatedly to (43) and using the chain rule we get the following Corollary to Proposition 25.

Corollary 26. Under the assumptions of Proposition 25, the following holds: For all $\alpha \in \mathbb{N}^{N}$, there exists a formal function $\Psi_{\alpha}: \mathbb{C}^{N} \times \mathbb{C}^{N} \times \mathbb{C}^{K\left(k_{0}+|\alpha|\right) N^{\prime}} \rightarrow$ $\mathbb{C}^{N^{\prime}}$ which is polynomial in its last $\left(K\left(k_{0}+|\alpha|\right)-K\left(k_{0}\right)\right) N^{\prime}$ entries such that

$$
\begin{aligned}
& \partial^{\alpha} H(Z) \\
- & \Psi_{\alpha}\left(Z, \zeta,\left(\partial^{\beta} \bar{H}(\zeta)-\partial^{\beta} \bar{H}(0)\right)_{0 \leq|\beta| \leq k_{0}},\left(\partial^{\beta} \bar{H}(\zeta)\right)_{k_{0}<|\beta| \leq k_{0}+|\alpha|}\right) \in I^{N^{\prime}}
\end{aligned}
$$

$\Psi_{\alpha}$ depends only on $M, M^{\prime}$, and on the values of $\partial^{\beta} H(0)$ for $|\beta| \leq k_{0}$, such that if $H^{\prime}: M \rightarrow M^{\prime}$ is another formal map with $\partial^{\beta} H(0)=\partial^{\beta} H^{\prime}(0)$ for $|\beta| \leq k_{0}$, then (47) holds with $H^{\prime}$ in place of $H$. If $M$ and $M^{\prime}$ are real-analytic, $\Psi_{\alpha}$ is convergent. If $M$ and $M^{\prime}$ are algebraic, so is $\Psi_{\alpha}$.

The next step is to repeatedly use (47) on the Segre sets. Recall (13a) and (14). Hence, choosing normal coordinates $Z=(z, w), \zeta=(\chi, \tau)$, we have that $f(z, 0,0,0)=0$ for all $f \in I$. Applying this fact to (47), we conclude that

$$
\partial^{\alpha} H(z, 0)=\Psi_{\alpha}\left(z, 0,0,0,0,\left(\partial^{\beta} \bar{H}(0)\right)_{k_{0}<|\beta| \leq k_{0}+|\alpha|}\right) .
$$

Note that the evaluation occuring causes no problems, since by Corollary 26, $\Psi_{\alpha}$ is a polynomial with respect to these variables. Hence the right hand side of (48) defines a formal map $\mathbb{C}^{n} \rightarrow \mathbb{C}^{N^{\prime}}$, is convergent if $M$ and $M^{\prime}$ are real-analytic, and algebraic, if $M$ and $M^{\prime}$ are algebraic. This is the case $k=0$ of the following Corollary (we are using the notation introduced before $(14))$ :

Corollary 27. For all $\alpha \in \mathbb{N}^{N}$, there exists a formal function $\Upsilon_{k, \alpha}: \mathbb{C}^{k n} \rightarrow$ $\mathbb{C}^{N^{\prime}}$ which depends only on $M, M^{\prime}$, and the derivatives $\partial^{\beta} H(0)$ for $|\beta| \leq$ $(k+1) k_{0}+|\alpha|$ such that

$$
\partial^{\alpha} H\left(v^{k+1}(z, \xi)\right)=\Upsilon_{k, \alpha}(z, \xi) .
$$

The dependence on the derivatives is as in Proposition 25: If $H^{\prime}: M \rightarrow M^{\prime}$ is another formal mapping with $\partial^{\beta} H(0)=\partial^{\beta} H^{\prime}(0)$ for $|\beta| \leq(k+1) k_{0}+|\alpha|$, then (49) holds with $H^{\prime}$ instead of $H$. If $M$ and $M^{\prime}$ are real-analytic, $\Upsilon_{k, \alpha}$ is convergent on a neighbourhood of $0 \in \mathbb{C}^{k n}$. If $M$ and $M^{\prime}$ are algebraic, so is $\Upsilon_{k, \alpha}$. 
Proof. We note that (48) is just the case $k=0$. We are doing induction on $k$. Assume the Corollary holds for $k<k^{\prime}$. By (47),

$$
\begin{aligned}
\partial^{\alpha} H\left(v^{k^{\prime}+1}\left(z^{\prime}, \xi^{\prime}\right)\right)= & \Psi_{\alpha}\left(v^{k^{\prime}+1}\left(z^{\prime}, \xi^{\prime}\right), \bar{v}^{k^{\prime}}\left(\xi^{\prime}\right),\left(\partial^{\beta} \bar{H}\left(\bar{v}^{k^{\prime}}\left(\xi^{\prime}\right)\right)\right.\right. \\
& \left.-\partial^{\beta} \bar{H}(0)\right)_{0 \leq|\beta| \leq k_{0}},\left(\partial^{\beta}\left(\bar{H}\left(\bar{v}^{k^{\prime}}\left(\xi^{\prime}\right)\right)\right)_{k_{0}<|\beta| \leq k_{0}+|\alpha|}\right) .
\end{aligned}
$$

Note that the compositions occuring on the right hand side are all welldefined. We now plug the induction hypothesis (49) for $k=k^{\prime}-1$ into (50). In fact, conjugating $(49)$ and replacing $(z, \xi)$ by $\left(\xi^{\prime}\right)$, we get that

$$
\partial^{\beta} \bar{H}\left(\bar{v}^{k^{\prime}}\left(\xi^{\prime}\right)\right)=\bar{\Upsilon}_{k^{\prime}-1, \beta}\left(\xi^{\prime}\right) .
$$

Now the highest order derivative we need is $|\beta|=k_{0}+|\alpha|$, which by assumption depends on the derivatives of $H$ of order up to $\left(k^{\prime}+2\right) k_{0}+|\alpha|$. This finishes the induction.

3.2. Proof of Theorems $\mathbf{1}, \mathbf{2}$, and $\mathbf{5}$. We start with Theorem 1 . We use Corollary 27 for $k=2 k_{1}-1$, where $k_{1}$ is the integer given by Theorem 10 . Since the manifolds are assumed to be real-analytic, $\Upsilon_{2 k_{1}, 0}$ is convergent in a neighbourhood of the origin. By Theorem 10, we can choose $\left(z_{0}, \xi_{0}\right)$ in this neighbourhood with $v^{2 k_{1}}\left(z_{0}, \xi_{0}\right)=0$ and such that the rank of $v^{2 k_{1}}$ is $N$ at $\left(z_{0}, \xi_{0}\right)$. As in the remark after Theorem 10, this implies that there is a holomorphic function $\psi$ defined in a neighbourhood of $0 \in \mathbb{C}^{N}$ such that $\psi(0)=\left(z_{0}, \xi_{0}\right)$ and $v^{2 k}(\psi(Z))=Z$. Hence,

$$
H(Z)=H\left(v^{2 k}(\psi(Z))\right)=\Upsilon_{2 k_{1}-1,0}(\psi(Z)) .
$$

Since the right hand side of (52) is convergent, so is the left hand side. This completes the proof of Theorem 1 .

Now assume that $H$ is $C^{\infty}-\mathrm{CR}$ and that $M$ and $M^{\prime}$ are smooth. Its associated formal holomorphic power series is then a formal holomorphic map between the formal submanifolds associated to $M$ and $M^{\prime}$ (see the remarks in 2.5). We use Corollary 27 for $k=k_{1}-1$, where $k_{1}$ is the integer given by Theorem 10 . Now set $K=k_{1}$. Then Corollary 27 implies that

$$
H\left(v^{k_{1}}(z, \xi)\right)=\Upsilon_{k_{1}-1,0}(z, \xi)=H^{\prime}\left(v^{k_{1}}(z, \xi)\right) .
$$

But $\operatorname{rk}\left(v^{k}\right)=N$, which by e.g., Proposition 5.3.5. in [5] implies that $H=H^{\prime}$ in the sense of equality of formal power series, which finishes the proof of Theorem 2.

Theorem 5 follows from Corollary 27 exactly like Theorem 1; we just note that it is enough to check that $H$ is algebraic on some open set $U$ containing the point $p_{0}$ where $H$ is assumed to be $k_{0}$-nondegenerate. 


\section{Levi-nondegenerate hypersurfaces. \\ The case $N^{\prime}=N+1$.}

In this section, we will assume that $M$ and $M^{\prime}$ are hypersurfaces, i.e., $d=$ $d^{\prime}=1$. In addition, we assume that they are Levi-nondegenerate (at our distinguished points). We start with a couple of general facts.

4.1. Levi-nondegeneracy. In normal coordinates, which we choose at our distinguished points $p_{0}$ and $p_{0}^{\prime}, M$ being Levi-nondegenerate means that we can assume

$$
Q_{z_{j} \chi_{k}}(0,0,0)=\delta_{j k} \epsilon_{k}, \quad 1 \leq k \leq n,
$$

where every $\epsilon_{k}$ is either $+i$ or $-i$, and likewise for $M^{\prime}$. Here is an easy technical result about the pullback of the Levi form by a map $H$ in normal coordinates, which we will use in the proof of Proposition 30.

Lemma 28. Let $M \subset \mathbb{C}^{N}, M^{\prime} \subset \mathbb{C}^{N^{\prime}}$ be given in normal coordinates by $w=Q(z, \bar{z}, \bar{w})$ and $w^{\prime}=Q\left(z^{\prime}, \bar{z}^{\prime}, \bar{w}^{\prime}\right)$, respectively. Assume that $H=(f, g)$ : $\left(\mathbb{C}^{N}, 0\right) \rightarrow\left(\mathbb{C}^{N^{\prime}}, 0\right)$ is a formal holomorphic map, and $H(M) \subset M^{\prime}$. Then

$$
g_{w}(0) Q_{z_{j} \bar{z}_{k}}(0)=\sum_{r, s=1}^{n^{\prime}} Q_{z_{r}^{\prime} \bar{z}_{s}^{\prime}}^{\prime}(0) f_{r z_{j}}(0) \overline{f_{s z_{k}}(0)}, \quad 1 \leq j, k \leq n .
$$

To prove this, set in $g(z, w)=Q^{\prime}(f(z, w), \bar{f}(\chi, \tau), \bar{g}(\chi, \tau)) \tau=0, w=$ $Q(z, \chi, 0)$ to obtain $g(z, Q(z, \chi, 0))=Q^{\prime}(f(z, Q(z, \chi, 0)), \bar{f}(\chi, 0), \bar{g}(\chi, 0))$. Differentiation with respect to $z_{j}$ and $\chi_{k}$ and evaluating at $z=\chi=0$ yields (55). This has the following consequence:

Corollary 29. Suppose that $M \subset \mathbb{C}^{N}$ and $M^{\prime} \subset \mathbb{C}^{N^{\prime}}$ are (formal) real hypersurfaces which are Levi-nondegenerate at $p_{0}$ and $p_{0}^{\prime}$, respectively, and $H:\left(\mathbb{C}^{N}, p_{0}\right) \rightarrow\left(\mathbb{C}^{N^{\prime}}, p_{0}^{\prime}\right)$ is a (formal) holomorphic map which takes $M$ into $M^{\prime}$ and is transversal at $p_{0}$. Then $H$ is immersive.

This is easy to see using normal coordinates (which we shall choose in a way such that $p=0$ and $\left.p^{\prime}=0\right)$. Since $g_{z^{\alpha}}(0)=0$, the differential of $H$ has the $=$ following form:

$$
\partial H(0)=\left[\begin{array}{cccc}
f_{1 z_{1}}(0) & \ldots & f_{1 z_{n}}(0) & f_{1 w}(0) \\
\vdots & & \vdots & \vdots \\
f_{n^{\prime} z_{1}}(0) & \ldots & f_{n^{\prime} z_{n}}(0) & f_{n^{\prime} w}(0) \\
0 & \ldots & 0 & g_{w}(0)
\end{array}\right]
$$

and $H$ is immersive if this matrix has rank $N$. Hence, if $H$ is transversal, $H$ is immersive if and only if the matrix

$$
\partial f(0)=\left[\begin{array}{ccc}
f_{1 z_{1}}(0) & \cdots & f_{1 z_{n}}(0) \\
\vdots & & \vdots \\
f_{n^{\prime} z_{1}}(0) & \cdots & f_{n^{\prime} z_{n}}(0)
\end{array}\right]
$$


has rank $n$. But by (55),

$$
g_{w}(0)\left(Q_{z_{j} \bar{z}_{k}}(0)\right)_{1 \leq j, k \leq n}=\overline{\partial f(0)}^{t}\left(Q_{z_{r}^{\prime} \bar{z}_{s}^{\prime}}^{\prime}(0)\right)_{1 \leq r, s \leq n^{\prime}} \partial f(0) .
$$

This implies that if $H$ is transversal, the rank of $\partial f(0)$ is at least $n$, which proves the corollary.

4.2. The basic identity for 1-degenerate maps. From now on we shall assume that $N^{\prime}=N+1$. Note that in the Levi-nondegenerate case,

$$
L_{k} Q_{z_{j}^{\prime}}(f(z, w), \overline{f(z, w)}, \overline{g(z, w)})(0)=\epsilon_{j}^{\prime} \overline{f_{j z_{k}}(0)}, \quad 1 \leq k \leq n, \quad 1 \leq j \leq n+1 .
$$

By Lemma 20, if $N^{\prime}=N+1$ and $H$ is transversal, the degeneracy $s$ of $H$ at $p_{0}$ is either 0 or 1 . In the case $s=0$, we can apply Theorem 1 and 2 to obtain Theorem 6 and 7 , since by Theorem 10 we see that $K \leq 2$ if the source manifold is a hypersurface. Hence, from now on we will assume that $s=1$. In this subsection, we will develop a basic identity for 1-degenerate maps between Levi-nondegenerate hypersurfaces. From (59) we see that in Lemma 16 we can choose $\alpha^{j}$ to be the multi-index with a 1 in the $i$-th spot and 0 elsewhere and reorder the $z^{\prime}$ 's, to get that after barring (26),

$$
\begin{aligned}
\bar{\Delta}(\chi, \tau) \bar{Q}_{\chi_{n+1}^{\prime}}^{\prime}(\bar{f}(\chi, \tau), f(z, w), g(z, w))- \\
\sum_{m=1}^{n} \bar{\Delta}_{m}(\chi, \tau) \bar{Q}_{\chi_{m}^{\prime}}^{\prime}(\bar{f}(\chi, \tau), f(z, w), g(z, w)) \in I,
\end{aligned}
$$

where now

$$
\begin{gathered}
\Delta(z, w)=\left|\begin{array}{ccc}
L_{1} Q_{z_{1}^{\prime}}^{\prime}(f, \bar{f}, \bar{g}) & \ldots & L_{1} Q_{z_{n}^{\prime}}^{\prime}(f, \bar{f}, \bar{g}) \\
\ldots & & \ldots \\
L_{n} Q_{z_{1}^{\prime}}^{\prime}(f, \bar{f}, \bar{g}) & \ldots & L_{n} Q_{z_{n}^{\prime}}^{\prime}(f, \bar{f}, \bar{g})
\end{array}\right|(z, w, 0, w), \\
\bar{\Delta}(0)=\left|\begin{array}{ccc}
\epsilon_{1}^{\prime} f_{1 z_{1}}(0) & \ldots & \epsilon_{n}^{\prime} f_{n z_{1}}(0) \\
\vdots & = & \vdots \\
\epsilon_{1}^{\prime} f_{1 z_{n}}(0) & \ldots & \epsilon_{n}^{\prime} f_{n z_{n}}(0)
\end{array}\right| \neq 0
\end{gathered}
$$

(63) $\Delta_{m}(z, w)=$

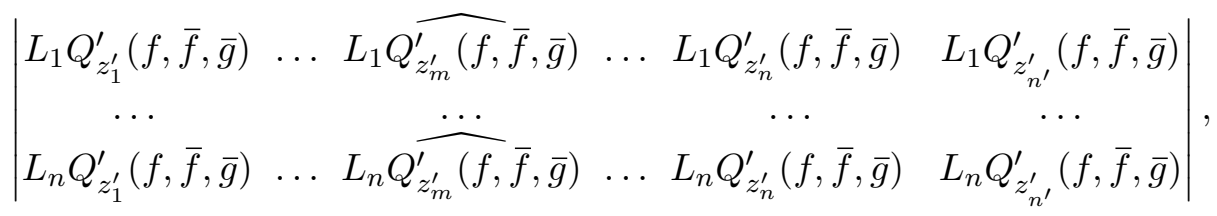


this matrix evaluated at $(z, w, 0, w)$,

$$
\bar{\Delta}_{m}(0)=(-1)^{n+m}\left|\begin{array}{ccccc}
\epsilon_{1}^{\prime} f_{1 z_{1}}(0) & \ldots & \epsilon_{m}^{\prime} \widehat{f_{m} z_{1}}(0) & \ldots & \epsilon_{n^{\prime}}^{\prime} f_{n^{\prime} z_{1}}(0) \\
\vdots & & \vdots & & \vdots \\
\epsilon_{1}^{\prime} f_{1 z_{n}}(0) & \ldots & \epsilon_{m}^{\prime} \widehat{f_{m} z_{n}}(0) & \ldots & \epsilon_{n^{\prime}}^{\prime} f_{n^{\prime} z_{n}}(0)
\end{array}\right|
$$

Since $H$ maps $M$ into $M^{\prime}$, the chain rule implies that we have formal functions $\Phi_{1}, \ldots, \Phi_{n}$ such that $\Phi_{j}(Z, \zeta, H(Z), \bar{H}(\zeta), \partial \bar{H}(\zeta))=L_{j} Q^{\prime}(f(z, w)$, $\bar{f}(\chi, \tau), \bar{g}(\chi, \tau))$ which are convergent if $M$ and $M^{\prime}$ are, and are polynomial in the derivatives of $\bar{H}$. As in the proof of Proposition 25 we obtain functions $\Phi_{j} \in \mathbb{C} \llbracket Z, \zeta, Z^{\prime}, \zeta^{\prime}, W \rrbracket$ where $W \in \mathbb{C}^{(N+1)^{2}}$, such that

$$
\Phi_{j}(Z, \zeta, H(Z), \bar{H}(\zeta), \partial \bar{H}(\zeta)-\partial \bar{H}(0)) \in I .
$$

From (60) we conclude that after a change of variables, we can write the function given there as $\Upsilon \in \mathbb{C} \llbracket Z, \zeta, Z^{\prime}, \zeta^{\prime}, T, U, W^{\prime} \rrbracket$ such that

$$
\Upsilon(Z, \zeta, H(Z), \bar{H}(\zeta), H(\zeta), H(0, \tau), \partial H(0, \tau)-\partial H(0)) \in I .
$$

$\Phi_{1}, \ldots, \Phi_{n}, \Upsilon$ only depend on $M, M^{\prime}$ and the derivative of $H$ evaluated at 0 , will agree for $H$ and $H^{\prime}$ with $\partial H(0)=\partial H^{\prime}(0)$, are convergent if $M$ and $M^{\prime}$ are real-analytic, and algebraic if $M$ and $M^{\prime}$ are algebraic. Consider the system of equations

$$
\begin{aligned}
& \Phi_{j}\left(Z, \zeta, Z^{\prime}, \zeta^{\prime}, W\right)=0, \quad 1 \leq j \leq n, \\
& \Upsilon\left(Z, \zeta, Z^{\prime}, \zeta^{\prime}, T, U, W^{\prime}\right)=0 \\
& Z_{N+1}^{\prime}=Q^{\prime}\left(Z_{1}^{\prime}, \ldots, Z_{N}^{\prime},=\zeta^{\prime}\right)
\end{aligned}
$$

in $\mathbb{C} \llbracket Z, \zeta, Z^{\prime}, \zeta^{\prime}, T, U, W, W^{\prime} \rrbracket$. We claim that we can apply the implicit function theorem to (67) to see that this system admits a unique solution in $Z^{\prime}$. In order to compute the Jacobian of (67) with respect to $Z^{\prime}$, first note that since $\Upsilon_{Z_{N+1}^{\prime}}(0,0,0,0,0)=0$ and $Z_{N+1}^{\prime}$ does not appear in any of the $\Phi_{j}$, it is enough to show that the determinant

$$
D=\left|\begin{array}{ccc}
\Phi_{1, Z_{1}^{\prime}}(0) & \ldots & \Phi_{1, Z_{N^{\prime}}^{\prime}}(0) \\
\vdots & & \vdots \\
\Phi_{n, Z_{1}^{\prime}}(0) & \ldots & \Phi_{n, Z_{N^{\prime}}^{\prime}}(0) \\
\Upsilon_{Z_{1}^{\prime}}(0) & \ldots & \Upsilon_{Z_{N^{\prime}}^{\prime}}(0)
\end{array}\right|
$$

is nonzero. Note that $\Phi_{k, Z_{j}^{\prime}}=\epsilon_{j}^{\prime} \overline{f_{j, z_{k}}(0)}$, that $\Upsilon_{Z_{j}^{\prime}}(0)=-\epsilon_{j}^{\prime} \bar{\Delta}_{j}(0)$ for $1 \leq j \leq n$ and $\Upsilon_{Z_{N}^{\prime}}(0)=\epsilon_{N}^{\prime} \bar{\Delta}(0)$. To simplify notation in the following argument, we write $\Delta(0)=-\Delta_{N}(0)$. Developing $D$ along the last row and 
using (62) and (64) we see that

$$
\begin{aligned}
& D= \pm\left(\epsilon_{1}^{\prime}\left|\begin{array}{ccc}
f_{2 z_{1}} & \ldots & f_{n^{\prime} z_{1}} \\
\vdots & & \vdots \\
f_{2 z_{n}} & \ldots & f_{n^{\prime} z_{n}}
\end{array}\right|\left|\begin{array}{ccc}
\overline{f_{2 z_{1}}} & \ldots & \overline{f_{n^{\prime} z_{1}}} \\
\vdots & & \vdots \\
\overline{f_{2 z_{n}}} & \ldots & \overline{f_{n^{\prime} z_{n}}}
\end{array}\right|\right. \\
& +\epsilon_{2}^{\prime}\left|\begin{array}{cccc}
f_{1 z_{1}} & f_{3 z_{1}} & \cdots & f_{n^{\prime} z_{1}} \\
\vdots & \vdots & & \vdots \\
f_{1 z_{n}} & f_{3 z_{n}} & \cdots & f_{n^{\prime} z_{n}}
\end{array}\right|\left|\begin{array}{cccc}
\overline{f_{1 z_{1}}} & \overline{f_{3 z_{1}}} & \cdots= & \overline{f_{n^{\prime} z_{1}}} \\
\vdots & \vdots & & \vdots= \\
\overline{f_{1 z_{n}}} & \overline{f_{3 z_{n}}} & \cdots= & \overline{f_{n^{\prime} z_{n}}}
\end{array}\right|+\ldots \\
& \left.\cdots+\epsilon_{n^{\prime}}^{\prime}\left|\begin{array}{cccc}
f_{1 z_{1}} & \cdots & f_{n-1 z_{1}} & f_{n z_{1}} \\
\vdots & & \vdots & \vdots \\
f_{1 z_{n}} & \ldots & f_{n-1 z_{n}} & f_{n z_{n}}
\end{array}\right|\left|\begin{array}{cccc}
\overline{f_{1 z_{1}}} & \ldots & \overline{f_{n-1 z_{1}}} & \overline{f_{n=z_{1}}} \\
\vdots & & \vdots & \vdots= \\
\overline{f_{1 z_{n}}} & \ldots & \overline{f_{n-1 z_{n}}} & \overline{f_{n=z_{n}}}
\end{array}\right|\right) .
\end{aligned}
$$

We apply the Cauchy-Binet Formula to (69) to see that $\pm D$ is equal to the determinant of

(70)

$$
\left[\begin{array}{cccc}
f_{1 z_{1}} & f_{2 z_{1}} & \ldots & f_{n^{\prime} z_{1}} \\
\vdots & \vdots & & \vdots \\
f_{1 z_{n}} & f_{2 z_{n}} & \ldots & f_{n^{\prime} z_{n}}
\end{array}\right]\left[\begin{array}{cccc}
\epsilon_{1}^{\prime} & 0 & \ldots & 0 \\
0 & \epsilon_{2}^{\prime} & \ldots & 0 \\
\vdots & \vdots & \ddots & \vdots \\
0 & 0 & \cdots & \epsilon_{n^{\prime}}^{\prime}
\end{array}\right]\left[\begin{array}{ccc}
\overline{f_{1 z_{1}}} & \ldots & \overline{f_{1 z_{n}}} \\
f_{2 z_{1}} & \ldots & \overline{f_{2 z_{n}}} \\
\vdots & & \vdots \\
\overline{f_{n^{\prime} z_{1}}} & \ldots & \overline{f_{n^{\prime} z_{n}}}
\end{array}\right]=A B C .
$$

The Cauchy-Binet formula tells us that in order to compute the determinant of this product, we just need to take the sum of the products of the determinants of square matrices obtained from $A$ and $B C$ by deleting a column in $A$ and the corresponding row in $B C$; but this sum is just the sum in (69). Now apply Lemma 28 to see that the determinant of (70) is just $\pm i g_{w}(0)$ which we assume to be nonzero. Hence, the claim is proved, and summarizing, we have proved the following.

Proposition 30. Let $M \subset \mathbb{C}^{N}$ and $M^{\prime} \subset \mathbb{C}^{N+1}$ be Levi-nondegenerate formal real hypersurfaces. Let $H: M \rightarrow M^{\prime}$ be a formal holomorphic map which is constantly 1-degenerate and transversal. Let $Z=(z, w), \zeta=(\chi, \tau)$ be normal coordinates for $M$. Then there exists a formal function $\Psi: \mathbb{C}^{N} \times$ $\mathbb{C}^{N} \times \mathbb{C}^{N+1} \times \mathbb{C}^{N+1} \times \mathbb{C}^{N(N+1)} \times \mathbb{C}^{N+1} \times \mathbb{C}^{N(N+1)} \rightarrow \mathbb{C}^{N+1}$ such that

$$
\begin{aligned}
& H(Z)-\Psi(Z, \zeta, \bar{H}(\zeta), H(\zeta), \partial \bar{H}(\zeta)-\partial \bar{H}(0), \\
& H(0, \tau), \partial H(0, \tau)-\partial H(0)) \in I^{N+1} .
\end{aligned}
$$

Furthermore, $\Psi$ depends only on $M, M^{\prime}$ and the first derivative of $H$ at 0 , such that if $H^{\prime}$ is another map fulfilling the assumptions of the proposition with $\partial H(0)=\partial H^{\prime}(0)$ then (71) holds with $H$ replaced by $H^{\prime}$. If $M$ and $M^{\prime}$ are real-analytic, $\Psi$ is convergent on a neighbourhood of the origin. If $M$ and $M^{\prime}$ are algebraic, so is $\Psi$. 
Differentiating this identity as in the proof of Corollary 26 we obtain the following.

Corollary 31. Under the assumptions of Proposition 30, the following holds: For all multi-indices $\alpha \in \mathbb{N}^{n}$, there is a formal function $\Psi_{\alpha}: \mathbb{C}^{N} \times \mathbb{C}^{N} \times$ $\mathbb{C}^{N+1} \times \mathbb{C}^{N+1} \times \mathbb{C}^{(K(1+|\alpha|)-1)(N+1)} \times \mathbb{C}^{K(|\alpha|)} \times \mathbb{C}^{N+1} \times \times \mathbb{C}^{(K(1+|\alpha|)-1)(N+1)} \rightarrow$ $\mathbb{C}^{N+1}$ which is polynomial in its 6 th, 7 th and 10 th variable such that

$$
\begin{aligned}
\partial^{\alpha} H(Z)- & \Psi_{\alpha}(Z, \zeta, \bar{H}(\zeta), H(\zeta), \\
& \partial \bar{H}(\zeta)-\partial \bar{H}(0),\left(\partial^{\beta} \bar{H}(\zeta)\right)_{2 \leq|\beta| \leq 1+|\alpha|},\left(\partial^{\beta} H(\zeta)\right)_{1 \leq|\beta| \leq|\alpha|}, \\
& \left.H(0, \tau), \partial H(0, \tau)-\partial H(0),\left(\partial^{\beta} H(0, \tau)\right)_{2 \leq|\beta| \leq 1+|\alpha|}\right) \in I^{N+1} ;
\end{aligned}
$$

$\Psi_{\alpha}$ depends only on $M, M^{\prime}$, and on the first derivative of $H$ at 0 such that if $H^{\prime}: M \rightarrow M^{\prime}$ is another formal map fulfilling the assumptions of Proposition 30 with $\partial H(0)=\partial H^{\prime}(0)$ then (72) holds with $H$ replaced by $H^{\prime}$. If $M$ and $M^{\prime}$ are real analytic, $\Psi_{\alpha}$ is convergent. If $M$ and $M^{\prime}$ are algebraic, so is $\Psi_{\alpha}$.

The main difference between (47) and (72) is that in (72) the argument $(0, \tau)$ appears. This means that we can only iterate (71) once, and hence we can determine $H$ from its 2 -jet at 0 only on the 2 nd Segre set. This is the main reason why we have to restrict to hypersurfaces here.

Corollary 32. Under the assumptions of Proposition 30, for all $\alpha \in \mathbb{N}^{N}$ there is a formal function $\Upsilon_{\alpha}: \mathbb{C}^{n} \times \mathbb{C}^{n} \rightarrow \mathbb{C}^{N+1}$ such that

$$
\partial^{\alpha} H(z, Q(z, \chi, 0))=\Upsilon_{\alpha}(z, \chi) .
$$

$\Upsilon_{\alpha}$ depends only on $M, M^{\prime}$ and $\partial^{\beta} H(0)$ for $|\beta| \leq 2+|\alpha|$ such that if $H^{\prime}$ is another map fulfilling the hypotheses of Proposition 30 with $\partial^{\beta} H(0)=$ $\partial^{\beta} H^{\prime}(0)$ for $|\beta| \leq 2+|\alpha|$, then (73) holds with $H$ replaced by $H^{\prime}$. If $M$ and $M^{\prime}$ are real-analytic, then $\Upsilon_{\alpha}$ is convergent on a neighbourhood of $0 \in$ $\mathbb{C}^{n} \times \mathbb{C}^{n}$. If $M$ and $M^{\prime}$ are algebraic, so is $\Upsilon_{\alpha}$.

The proof of this corollary is by induction just as in Corollary 26 and left to the reader. Theorem 7 in the case $s=1$ follows from Corollary 32 just as Theorem 2 follows from Corollary 26. Theorem 9 also follows easily from Corollary 32 since by Lemma 22 we can always pass to a point where $H$ is of constant degeneracy. However, since we can only work on the second Segre set, we have to work a little harder for Theorem 6 . We are basically following an argument given in [2].

4.3. Proof of Theorem 6. From Corollary 32 we conclude that

$$
H(z, w)=\sum_{j=1}^{\infty} H^{j}(z) w^{j}
$$


where $H^{j}(z)=\frac{1}{j !} H_{w^{j}}(z, 0)$ is convergent. We now want to solve the equation $w=Q(z, \chi, 0)$ for $\chi$ in $w$. Choose a $\chi_{0}$ such that the function $\phi(z, t)=$ $Q\left(z, \chi_{0} t, 0\right)$, which is defined in a neighbourhood of the origin in $\mathbb{C}^{n} \times \mathbb{C}$, has a derivative in $t$ which is not constantly vanishing. We write

$$
\phi(z, t)=\sum_{j=1}^{\infty} \phi_{j}(z) t^{j}
$$

and define a convergent power series $\psi(z, t)=t+\sum_{j=2}^{\infty} C_{j}(z) t^{j}$ where $C_{j}(z)=\phi_{j}(z) \phi_{1}(z)^{j-2}$ for $j \geq 2$. By the implicit function theorem, the equation $w=\psi(z, t)$ has a solution $t=\theta(z, w)$ which is convergent in a neighbourhood of the origin in $\mathbb{C}^{n} \times \mathbb{C}$. Then $t=\phi_{1}(z) \theta\left(z, \frac{w}{\phi_{1}(z)^{2}}\right)$ solves $w=Q\left(z, \chi_{0} t, 0\right)$. By changing $\theta$, we can assume that $\phi_{1}(z)=A(z)$ is a Weierstrass polynomial. Hence we conclude that

$$
H(z, w)=F\left(z, \frac{w}{A(z)^{2}}\right)
$$

where $F$ is now a function which is convergent in a neighbourhood of the origin in $\mathbb{C}^{N}$. We expand $F$ in the following way: $F(z, t)=\sum_{j=1}^{\infty} F_{j}(z) t^{j}$. Comparing coefficients in (76), we conclude that $H_{j}(z)=F_{j}(z) A(z)^{-2 j}$. We now apply the division theorem to see that

$$
F_{j}(z)=B_{j}(z) A(z)^{2 j}+r_{j}(z)
$$

where $r_{j}(z)$ is a $\left(\mathbb{C}^{N+1}\right.$-valued) Weierstrass polynomial of degree less than $2 j p$ where $p$ is the degree of the Weierstrass polynomial $A(z)$. Furthermore, we have the inequality

$$
\left\|B_{j}(z)\right\| \leq C^{j}\left\|F_{j}(z)\right\|
$$

which holds for $z$ in a neighbourhood of the origin, with some constant $C$ (see e.g., [10], Theorem 6.1.1.). Since $H_{j}$ is convergent, we conclude that $r_{j}$ is the zero polynomial. So $H_{j}(z)=B_{j}(z)$ and from (78) we finally conclude that $H(z, w)$ is convergent in a neighbourhood of the origin.

\section{Strictly pseudoconvex targets.}

We will just indicate how to derive a basic identity in this case; the proof is then finished by exactly the same arguments as in the Levi-nondegenerate case. By the Chern-Moser normal form ([8]), we can in particular assume that the target hypersurface is given in normal coordinates $\left(z^{\prime}, w^{\prime}\right)$ by $w^{\prime}=$ $Q^{\prime}\left(z^{\prime}, \chi^{\prime}, \tau^{\prime}\right)$, where

$$
Q^{\prime}\left(z^{\prime}, \chi^{\prime}, \tau^{\prime}\right)=\tau+i\left\langle z^{\prime}, \chi^{\prime}\right\rangle+\sum_{\substack{\alpha, \beta, \gamma \\|\alpha|,|\beta| \geq 2}} c_{\alpha, \beta, \gamma} z^{\prime \alpha} \chi^{\prime \beta} \tau^{\prime \gamma} .
$$


In this equation, $\left\langle z^{\prime}, \chi^{\prime}\right\rangle=\sum_{j=1}^{n^{\prime}} z_{j}^{\prime} \chi_{j}^{\prime}=\left(z^{\prime}\right)^{t} \chi^{\prime}$. It follows that

$$
\left.L^{\alpha} Q_{Z^{\prime}}^{\prime}(f(z, w), \bar{f}(\chi, \tau), \bar{g}(\chi, \tau))\right|_{0}=L^{\alpha} \bar{f}(0) .
$$

We only needed the Chern-Moser normal form in order to get rid of terms of the form $z_{j}^{\prime} \chi^{\prime \beta}$ in the power series expansion for $Q^{\prime}$ where $|\beta|>1$ in order to arrive at this easy formula for $\left.L^{\alpha} Q_{Z^{\prime}}^{\prime}(f(z, w), \bar{f}(\chi, \tau), \bar{g}(\chi, \tau))\right|_{0}$. Hence, if $H$ is constantly $\left(k_{0}, s\right)$ degenerate at 0 , we can choose $t=n^{\prime}-s$ multiindices $\alpha^{1}, \ldots, \alpha^{t}, \alpha^{j} \in \mathbb{N}^{n},\left|\alpha^{j}\right| \leq k_{0}$, such that the vectors $\xi_{j}=L^{\alpha^{j}} \bar{f}(0)$, $1 \leq j \leq t$ are linearly independent in $\mathbb{C}^{n^{\prime}}$. We extend this set to a basis $\xi_{1}, \ldots, \xi_{n^{\prime}}$ of $\mathbb{C}^{n^{\prime}}$. Since $M^{\prime}$ is strictly pseudoconvex, we can use the GramSchmidt orthogonalization process to obtain vectors $v_{1}, \ldots, v_{n^{\prime}}$ which are orthonormal with respect to the standard hermitian product on $\mathbb{C}^{n^{\prime}}$, and a lower triangular invertible matrix $C$ such that $V=C E$, where $V$ denotes the unitary matrix with rows $v_{1}, \ldots, v_{n^{\prime}}$, and $E$ denotes the matrix with rows $\xi_{1}, \ldots, \xi_{n^{\prime}}$. We change coordinates by $\widetilde{z}=V z, \widetilde{w}=w$. Since $V$ is unitary, the defining function still has the form (79); in particular, (80) holds with $f$ replaced by $\tilde{f}$, and $L^{\alpha} \overline{\widetilde{f}(0)}=\bar{V} L^{\alpha} \bar{f}(0)$. Since $\bar{V} E=\left(C^{t}\right)^{-} 1$ is upper triangular, it follows that we can assume $\xi_{j}^{k}=\left(L^{\alpha^{j}} \bar{f}(0)\right)_{k}=0$ for $j>k$. Note that the same change of coordinates works for every other map whose $k_{0}$-jet at the origin agrees with the $k_{0}$-jet of $H$, if $k_{0}>1$. Note that we have used the strict pseudoconvexity only to reduce to the case where the matrix $\left(L^{\alpha^{j}} \bar{f}_{k}(0)\right)$ is triangular.

We now start as in the proof of Proposition 25 and obtain formal functions $\Phi_{j}\left(Z, \zeta, Z^{\prime}, \zeta^{\prime}, W\right) \in \mathbb{C} \llbracket Z, \zeta, Z^{\prime}, \zeta^{\prime}, W \rrbracket$, defined by $\Phi_{j}(Z, \zeta, H(Z), \bar{H}(\zeta)$, $\left.\left(\partial^{\beta} \bar{H}(\zeta)-\partial^{\beta} \bar{H}(0)\right)_{|\beta| \leq k_{0}}\right)=\left(L^{\alpha^{j}} Q^{\prime}(f, \bar{f}, \bar{g})\right)(Z, \zeta)$, which are convergent if $M$ and $M^{\prime}$ are real-analytic and algebraic if $M$ and $M^{\prime}$ are algebraic. Also note that $\Phi_{j}$ does not depend on $Z_{N^{\prime}}^{\prime}$.

The missing equations are supplied by Lemma 16 . So we define formal functions $\Upsilon_{k}\left(Z, \zeta, Z, \zeta^{\prime}, S, T, W^{\prime}\right), 1 \leq k \leq s$, by

$$
\begin{aligned}
& \Upsilon_{j-t}\left(Z, \zeta, H(Z), \bar{H}(\zeta), H(\zeta), H(0, \tau),\left(\partial^{\beta} H(0, \tau)-\partial^{\beta} H(0)\right)_{1 \leq|\beta| \leq k_{0}}\right) \\
& =\bar{\Delta}(\chi, \tau) \bar{Q}_{\chi_{j}^{\prime}}(\bar{f}(\chi, \tau), f(z, w), g(z, w)) \\
& \quad-\sum_{m=1}^{t} \bar{\Delta}_{m}(\chi, \tau) \bar{Q}_{\chi_{m}^{\prime}}(\bar{f}(\chi, \tau), f(z, w), g(z, w))
\end{aligned}
$$

for $t+1 \leq j \leq n^{\prime}$, where $\bar{\Delta}$ is defined by (27) and $\bar{\Delta}_{m}$ is given by (28). Recall that the functions $\Upsilon_{k}$ are convergent if $M$ and $M^{\prime}$ are real-analytic and algebraic if $M$ and $M^{\prime}$ are algebraic. We now claim that we can apply 
the implicit function theorem to solve the system

$$
\begin{aligned}
& \Phi_{j}\left(Z, \zeta, Z^{\prime}, \zeta^{\prime}, W\right)=0, \quad 1 \leq j \leq t, \\
& \Upsilon_{k}\left(Z, \zeta, Z^{\prime}, \zeta^{\prime}, S, T, W^{\prime}\right)=0, \quad 1 \leq k \leq s, \\
& Z_{N^{\prime}}^{\prime}=Q^{\prime}\left(Z_{1}^{\prime}, \ldots, Z_{n^{\prime}}^{\prime}, \zeta^{\prime}\right),
\end{aligned}
$$

uniquely in $Z^{\prime}$. First note that $\Phi_{j, Z_{N^{\prime}}^{\prime}}(0)=\Upsilon_{k, Z_{N^{\prime}}^{\prime}}(0)=0$ for $1 \leq j \leq t$, and $1 \leq k \leq s$. So we only need to consider the Jacobian of $\left(\Phi_{1}, \ldots, \Phi_{t}, \Upsilon_{1}, \ldots\right.$, $\left.\Upsilon_{s}\right)$ with respect to $\left(Z_{1}^{\prime}, \ldots, Z_{n^{\prime}}^{\prime}\right)$. Now $\Phi_{j, Z^{\prime}}(0)=\xi_{j}$, and a little computation shows that $\Upsilon_{k, Z^{\prime}}(0)=\left(\bar{\Delta}_{1}(0), \ldots, \bar{\Delta}_{t}(0), 0, \ldots \bar{\Delta}(0), \ldots, 0\right)$, where the $\Delta(0)$ appears in the $(t+k)$-th spot, $1 \leq k \leq s$. Recall that $\xi_{j}^{k}=0, j>k$, by our choice of coordinates. Writing out the determinant we see that indeed the implicit function theorem applies. This gives the desired basic identity.

\section{References}

[1] M.S. Baouendi, P. Ebenfelt and L.P. Rothschild, Algebraicity of holomorphic mappings between real algebraic sets in $\mathbb{C}^{n}$, Acta Math., 177(2) (1996), 225-273, MR 99b:32030, Zbl 890.32005.

[2] _ Parametrization of local biholomorphisms of real analytic hypersurfaces, Asian J. Math., 1(1) (1997), 1-16, MR 99b:32022, Zbl 943.32021.

[3] _ Convergence and finite determination for formal CR mappings, J. Amer. Math. Soc., 13 (2000), 697-723, CMP 1775734.

[4] - Rational dependence of smooth and analytic CR mappings on their jets, Math. Ann., 315 (1999), 205-249, MR 2001b:32075, Zbl 942.32027.

[5] _ Real Submanifolds in Complex Space and their Mappings, Princeton University Press, Princeton, NJ, 1999, MR 2000b:32066, Zbl 944.32040.

[6] M.S. Baouendi, X. Huang and L.P. Rothschild, Regularity of CR mappings between algebraic hypersurfaces, Invent. Math., 125(1) (1996), 13-36, MR 97c:32028, Zbl 855.32009.

[7] M.S. Baouendi and L.P. Rothschild, Mappings of real algebraic hypersurfaces, J. Amer. Math. Soc., 8(4) (1995), 997-1015, MR 96f:32039, Zbl 869.14025.

[8] S.S. Chern and J.K. Moser, Real hypersurfaces in complex manifolds, Acta Math., 133 (1974), 219-271, MR 54 \#13112, Zbl 302.32015.

[9] J.P. D'Angelo, Real hypersurfaces, orders of contact, and applications, Ann. of Math. (2), 115(3) (1982), 615-637, MR 84a:32027, Zbl 488.32008.

[10] L. Hörmander, An Introduction to Complex Analysis in Several Variables, NorthHolland, Amsterdam-London-New York-Tokyo, 1990, MR 91a:32001, Zbl 685.32001.

[11] X. Huang, On the mapping problem for algebraic real hypersurfaces in the complex spaces of different dimensions, Ann. Inst. Fourier (Grenoble), 44(2) (1994), 433-463, MR 95i:32030, Zbl 803.32011.

[12] B. Lamel, A reflection principle for real-analytic submanifolds of complex spaces, preprint, 1-7, math.CV/9904118, 1999. 
[13] N. Mir, Germs of holomorphic mappings between real algebraic hypersurfaces, Ann. Inst. Fourier (Grenoble), 48(4) (1998), 1025-1043, MR 2000c:32059, Zbl 914.32009.

[14] R. Sharipov and A. Sukhov, On CR mappings between algebraic Cauchy-Riemann manifolds and separate analyticity, Trans. Am. Math. Soc., 348(2) (1996), 767-780, MR 96g:32019, Zbl 851.32017.

[15] N.K. Stanton, Infinitesimal CR automorphisms of real hypersurfaces, Amer. J. Math., 118(1) (1996), 209-233, MR 97h:32027, Zbl 849.32012.

[16] A. Sukhov, On algebraicity of complex analytic sets, Math. USSR Sbornik, 74 (1993), 419-426.

[17] S.M. Webster, On the mapping problem for algebraic real hypersurfaces, Invent. Math., 43(1) (1977), 53-68, MR 57 \#3431, Zbl 355.32026.

[18] D. Zaitsev, Algebraicity of local holomorphisms between real-algebraic submanifolds of complex spaces, Acta Math., 183(2) (1999), 273-305, MR 2001a:32024.

Received November 9, 1999 and revised June 16, 2000.

Department of Mathematics, 0112

University of California, San Diego

SAN Diego, CA 92092-0112

E-mail address: blamel@euclid.ucsd.edu 